Part of Journal of Research of the National Bureau of Standards

\title{
Preliminary Description and Analysis of the First Spectrum of Uranium
}

\author{
By C. C. Kiess, Curtis J. Humphreys, and Donald D. Laun
}

\begin{abstract}
Observations of the spectra emitted by uranium under are and spark excitation have afforded a separation of the lines of neutral atoms from those emitted by ions. A list of more than 9,000 lines with accurate wavelengths, wave numbers, and estimated intensities, for the region $2900 \mathrm{~A}$ to $11000 \mathrm{~A}$, has been compiled as descriptive of the spectrum of the neutral atom. About 2,000 of these lines have been classified as combinations between 18 low and metastable odd energy levels with about 280 high even levels. Well-resolved Zeeman patterns give $g$-values for several low levels that identify them as components of ${ }^{5} \mathrm{~L},{ }^{5} \mathrm{~K},{ }^{7} \mathrm{M}$, and ${ }^{7} \mathrm{~K}$ terms arising in the electron configurations $f^{3} d s^{2}$ and $f^{3} d^{2} s$. The spectrum of uranium is interpreted as that of a rare-earth element analogous to neodymium, uranium being the third member of a second group of rare earths beginning with thorium. From the fact that uranium is easily ionized in electric arcs and magnetic fields, and also that the short-wave limit of the observed spectrum does not extend below $2900 \mathrm{~A}$, it is concluded that the ionization potential of neutral uranium atoms is approximately 4 volts.
\end{abstract}

\section{Introduction}

In the more than 30 years that have elapsed since the appearance, in 1912, of volume 6 of Kayser's "Handbuch der Spectroscopie", only a few additions have been made to our knowledge of the spectra emitted by uranium atoms. At that time Professor Kayser's [1] ${ }^{1}$ description of the uranium spectrum was expressed in the words: "Es gehört zu den linienreichsten Spectren der Elemente, gleichzeitig zu den am wenigsten charakteristischen. ... Der Unterschied zwis-

\footnotetext{
1 Figures in brackets indicate the literature references at the end of this paper.
}

chen Bogen und Funken ist ausserordentlich gering, wenn überhaupt vorhanden; . . . ." In 1916 Meissner [2] published a list of 17 infrared uranium lines, and in 1920 Kiess and Meggers [3] published a list of more than 700 lines in the range

\section{Contents}

I. Introduction

II. Experimental procedure

III. Results _._.

IV. References ............. 72 
from $5500 \mathrm{~A}$, in the visible spectrum, to beyond $9500 \mathrm{~A}$, in the infrared. But these extensions of the spectrum, and the improvements in wavelengths and intensities reported for uranium lines in the MIT Wavelength Tables [4], did not much alter Kayser's appraisal, as stated above.

In order to set up a system of energy levels for neutral and ionized uranium atoms, it is desirable to have a complete description of the spectrum emitted by each species of atom. Such a description includes not only precise wavelengths and well-estimated intensities for the lines, but also other characteristics, such as reversibility, diffuseness, Zeeman effect, etc. All the published descriptions of uranium spectra agree in reporting a continuous background on which the line spectra are superimposed. The effect of this continuum, in which the fainter and diffuse lines are completely lost, is to attenuate the intensity differences between the measurable lines. Despite this unfavorable circumstance, however, an enormous number of lines, in excess of 5,000 , have been cataloged for uranium for both arc and spark excitation. No other significant descriptive data appear to have been published. The pressure shifts measured by W. J. Humphreys [5], and the reversals observed by McLennan, Cohen, and Liggett [6], apparently refer to lines emitted by other than uranium atoms.

Late in 1942 the Spectroscopy Section of the National Bureau of Standards was requested to undertake an investigation of the uranium spectra and to determine, if possible, the system of energy levels responsible for their production. Available for the investigation were several spectrographs, and such auxiliary equipment as interferometers, electromagnet, underwater spark, etc. Highly purified uranium metal was also available for use as electrode material. Accordingly, a program of work was mapped out to observe the spectra as emitted by arcs and condensed spark discharges in air at normal and at reduced pressures, and by discharges in hollow cathodes, in underwater sparks, and in magnetic fields.

\section{Experimental Procedure}

Five spectrographs in all were employed in making the Bureau of Standards observations. For the region between $2100 \mathrm{~A}$ and $2800 \mathrm{~A}$, in the ultraviolet, a large quartz-prism spectrograph by Hilger was used. The dispersion of this instrument, described elsewhere [7], varies from 0.4 to $1.0 \mathrm{~A}$ per millimeter in the region for which it was used. A spectrograph in which the dispersive piece was a grating, ruled 30,000 lines per inch by R. W. Wood [8], was used in the first order to record the spectra from $2000 \mathrm{~A}$ to $3000 \mathrm{~A}$, in the ultraviolet, and from $4200 \mathrm{~A}$ to $8500 \mathrm{~A}$ in the visible and infrared, with a dispersion of approximately $2 \mathrm{~A}$ per millimeter. In the second order, dispersion of $1 \mathrm{~A}$ per millimeter, it was used to record the spectra between $2600 \mathrm{~A}$ and $4300 \mathrm{~A}$. A second grating with 15,000 lines per inch, also ruled by Wood, was used in the first order for recording the red and infrared portions of the spectra between $7000 \mathrm{~A}$ and $10000 \mathrm{~A}$, the dispersion being $5 \mathrm{~A}$ per millimeter. The grating ruled by J. A. Anderson with 7,500 lines per inch was used to photograph the region from $9000 \mathrm{~A}$ to beyond $11000 \mathrm{~A}$, in the first order. These three gratings are concave gratings of approximately 21-foot radius of curvature and are mounted in parallel light, according to the method of Wadsworth, as described by Meggers and Burns [9]. A fourth concave grating, ruled by Wood with 15,000 lines per inch, and set up in an Eagle mounting, was used in the fourth order for the region $4100 \mathrm{~A}$ to $5000 \mathrm{~A}$, the dispersion being $1.2 \mathrm{~A}$ per millimeter.

The electrodes used in the arcs and sparks were rods of uranium metal of highest purity. The arcs were operated on currents of 5 to 7 amperes supplied by a 220 -volt circuit. The condensed sparks were obtained from condensers that were charged from the 50,000-volt side of an induction coil whose primary carried a current of 10 amperes from a 110-volt a-c circuit. Each condenser unit was rated at 0.002 microfarad, so that when three or five were connected in parallel the capacitance of the discharge circuit was 0.006 or 0.010 microfarad. No self-inductance was used in this circuit.

The first observations made with arcs and sparks in air revealed the dark background reported by the earlier investigators. This background was found to emanate not only from the white-hot tips of the electrodes but also, and in greater intensity, from the incandescent particles 
of uranium oxide that are formed in the outer envelopes surrounding the excited atoms. Various devices were tried to minimize, if not eliminate, the background effect. None was entirely successful, but satisfactory results were obtained either with the source shielded so that light from only a narrow zone between the electrodes could pass to the slit of the spectrograph, or with the source in a partially evacuated enclosure. The procedure finally adopted in making the spectrograms was to expose the photographic plates in succession to the shielded, or enclosed, uranium arc, to the uranium spark in air, to a similar spark enclosed in air at reduced pressure, and to the iron arc in air for the reference standards. The different exposures were recorded so that each exposure to a uranium source stood in juxtaposition to an iron exposure.

Inspection of the spectrograms showed that the number of lines recorded on them for any region was at least twice as great as hitherto reported. Yet the fact that arc and spark spectra were adjacent to each other on the plates made it possible to separate them according to the stage of ionization of their emitters on the basis of estimated intensities alone. Nevertheless, it was deemed desirable to carry out experiments on the reversal of the spectrum to single out the lines characteristic of neutral atoms and those most likely to originate in their lowest energy states. For this phase of the investigation the underwater spark was adopted, as described by Meggers and Laporte [10]. Although the continuum from the source, a condensed spark discharge between uranium electrodes submerged in flowing distilled water, was recorded on the spectrograms from the ultraviolet to the near infrared, yet no absorptions attributable to uranium atoms were observed. Instead, a few of the stronger emission lines of singly ionized uranium atoms appeared, superimposed on the continuous background.

The Zeeman-effect observations were made with a large, water-cooled Weiss magnet, equipped with both iron and ferro-cobalt pole pieces. With currents of 160 amperes applied to the coils, fields ranging in strength from 28,000 to 35,000 oersteds were obtained for pole gaps between 8 and 5 millimeters. In the earlier observations the source was a condensed spark discharge between electrodes cut from wire about a millimeter in diameter and accurately centered in the pole gap of the magnet. This source favored the excitation of lines from uranium ions rather than those from neutral atoms and was replaced by an a-c arc in which a current of 1 ampere was carried under an electromotive force of 2,200 volts. Subsequently, this source was replaced by a Back [11] lamp in which an interrupted arc was operated on 1 ampere at 220 volts, the air pressure within the arc enclosure being reduced to oneeighth or one-tenth atmosphere. This source proved to be the best for excitation of lines of the neutral atom and was used in a series of observations covering the range from $3000 \mathrm{~A}$ to beyond $9000 \mathrm{~A}$. In order to calibrate the fields, exposures were also made with electrodes of copper, silver, magnesium, and other elements, to secure resolved magnetic patterns of known separations.

Between the pole gap of the magnet and the condensing lens of the spectrograph was placed a large quartz Wollaston prism, which separated the two polarizations in the Zeeman pattern vertically on the spectrograph slit. Owing to the stigmatic properties of a grating mounted in parallel light, it was thus possible to photograph simultaneously the two polarizations. Between them an occulting bar over the slit reserved an unexposed strip on the photographic plates for recording the spectrum without the magnetic field. The no-field exposures served effectively to mark the centers of the magnetic patterns, a desideratum of utmost importance in a complex spectrum where overlapping of the components in the patterns of close lines is of frequent occurrence.

\section{Results}

The spectrograms from which were derived the results presented below were measured and reduced according to well-known procedures. All the wavelength measurements are referred to iron lines adopted as international secondary standards or for which wavelengths determined by inter- ference measurements are available. The wavelengths adopted for the uranium lines are the means of two or more determinations from measurements on separate spectrograms. The intensities assigned to the lines are estimates on an expanded scale ranging from 0 or 1 for the faintest 
lines measured to 500 or more for the strongest. Most of the lines are sharp, with no tendency toward diffuseness nor any indication of self-reversal.

In the final tabulation of the results of the measurements a list has been compiled of more than 9,000 wavelengths that are believed to be characteristic of the spectrum emitted by neutral uranium atoms. The list covers the range $2900 \mathrm{~A}$ in the ultraviolet to $11000 \mathrm{~A}$ in the infrared, the basis of selection for the lines being their relative intensities in the spectra of arc and spark. That this method of selection presented no great difficulty may be seen in figure 1 , which shows a portion of the violet region. For some lines, however, the ionic origin is definitely established by the number of $p$-components that they exhibit in well-resolved Zeeman patterns. For Ur, which is built up on levels of odd multiplicity, this number is odd, whereas for U Ir it is even.

The Zeeman effects observed for most of the lines are either triplets or unresolved patterns. However, well-resolved patterns were recorded for a small number of lines. It was these that gave the clue to the analysis of the spectrum by telling uniquely the inner quantum numbers and $g$-values of the levels involved in the production of the lines. But to interpret these $g$-values and inner quantum numbers, it was first necessary to extend existing tables of theoretical Zeeman effects to cover terms of bigher $L$-value. In table 1 are given a few lines with their Zeeman patterns, of which some are illustrated in figure 2.

TABLE 1.-Zeeman effects of some U I lines

\begin{tabular}{|c|c|}
\hline $\begin{array}{l}\text { Wave- } \\
\text { length }\end{array}$ & Observed Zeeman effect \\
\hline 8381.86 & $(0.00,0.26,0.53,0.80,1.07,1.30)$ \\
\hline 8223. 08 & $(0.00,0.20,0.40,0.60,0.81,1.04)$ \\
\hline 7101. 61 & $(0.00, .012,0.23,0.34) . . \quad .0 .80,0.93$ \\
\hline 6820. 76 & $(0.00,0.23,0.47,0.71,0.97,1.17)$ i. $^{\circ}$ \\
\hline 6395. 42 & $(0.00,0.15,0.32,0.48,0.63,0.81,0.94)$ \\
\hline 5997.31 & $(0.00,0.33,0.68,1.04,1.32,1.69,2.06)$ \\
\hline 5971.50 & $\begin{array}{r}(0.00 .0 .15,0.29,0.45,0.58,0.73) \\
1.18,1.34,1.50,1.64\end{array}$ \\
\hline
\end{tabular}

With the key to the structure of the spectrum thus in hand, a search through the tabulated wave numbers of the lines brought to light numerous recurring constant differences, of which some proved to be significant. Although laborious, this method of search proved fruitful in leading to the groups of odd and even energy levels presented in tables 2 and 3. The odd levels have been assigned their designations on the basis of $g$-values derived from the resolved Zeeman-effect observations. Such terms can arise only from electron configurations containing $f$-electrons. There are various possible groupings of the six uranium valence electrons into such configurations, but the ${ }^{7} \mathrm{M}$ term can arise only in the configuration $f^{3} d^{2} s$. Inasmuch as the low ${ }^{5} \mathrm{~L}$ and ${ }^{5} \mathrm{~K}$ terms are of the same parity as ${ }^{7} \mathrm{M}$ and are unaccompanied by corresponding terms of the septet system, it is necessary to conclude that they owe their origin to the configuration $f^{3} d s^{2}$ rather than to some other configuration.

\begin{tabular}{|c|c|r|r|r|}
\multicolumn{5}{|c|}{ TABLE 2.-Low odd levels of $\mathrm{U}_{\mathrm{I}}$} \\
\hline \hline $\begin{array}{c}\text { Electron } \\
\text { configura- } \\
\text { tion }\end{array}$ & Term symbol & Term value & \multicolumn{2}{|c|}{$g$-values } \\
\hline & & & LS & Observed \\
\hline$f^{3} d s^{2}$ & ${ }^{5} \mathrm{~L}_{6}^{\circ}$ & 0.0 & 0.714 & 0.75 \\
$f^{3} d s^{2}$ & ${ }^{5} \mathrm{~K}_{5}^{\circ}$ & 620.3 & .667 & .73 \\
& $\mathrm{~A}_{7}^{\circ}={ }^{5} \mathrm{~L}_{7}^{\circ} ?$ & 3800.8 & & \\
& $\mathrm{~A}_{3}^{\circ}$ & 3868.4 & & \\
$f^{3} d s^{2}$ & ${ }^{5} \mathrm{~K}_{6}^{\circ}$ & 4275.7 & .905 & .93 \\
& ${ }^{5} \mathrm{I}_{4}^{\circ}$ & 4453.4 & .600 & .66 \\
$f^{3} d^{2} s$ & ${ }^{7} \mathrm{~K}_{5}^{\circ}$ & 5762.0 & .767 & .82 \\
& ${ }^{5} \mathrm{H}_{4}^{\circ}$ & 5991.3 & .900 & .86 \\
$f^{3} d^{2} s$ & ${ }^{7} \mathrm{M}_{6}^{\circ}$ & 6249.0 & .571 & .62 \\
$f^{3} d^{2} s$ & ${ }^{7} \mathrm{~K}_{6}^{\circ}$ & 7005.5 & .976 & .97 \\
& $\mathrm{~B}_{3}^{\circ}$ & 7103.9 & & \\
& $\mathrm{~B}_{7}^{\circ}={ }^{5} \mathrm{~K}_{7}^{\circ} ?$ & 7326.1 & & \\
& $\mathrm{~A}_{8}^{\circ}={ }^{5} \mathrm{~L}_{8}^{\circ} ?$ & 7645.6 & & \\
& $\mathrm{C}_{7}^{\circ}={ }^{7} \mathrm{M}_{7}^{\circ} ?$ & 8118.6 & & \\
& $\mathrm{D}_{7}^{\circ}={ }^{7} \mathrm{~K}_{7}^{\circ} ?$ & 10347.3 & & \\
& $\mathrm{~B}_{8}^{\circ}={ }^{5} \mathrm{~K}_{8}^{\circ} ?$ & 10685.7 & & \\
& $\mathrm{~A}_{5}^{\circ}$ & 11545.4 & & \\
& $\mathrm{~A}_{9}^{\circ}$ & 13127.9 & & \\
& & &
\end{tabular}

This conclusion is further supported by the interpretation of the spectrum Th II due to singly ionized thorium atoms. This spectrum has been analyzed independently by two groups of investigators [12], who are in agreement in finding that although the lowest energy states belong to the $d s^{2}, d^{2} s$, and $d^{3}$ configurations, yet an energetically prominent set of low states is also present due to the configurations $f s^{2}$ and $f d s$. In passing from thorium to uranium, we might expect, on the assumption that the binding of $f$-type electrons is preferred, the configuration $f^{3} d s$ to give the lowest states of the ion, and $f^{3} d s^{2}$ and $f^{3} d^{2} s$ the lowest and metastable states of the neutral uranium atom. ${ }^{2}$

In a paper just received here (Physica 11,$419 ; 1946)$ Schuurmans in reporting the lowest levels of U II assigns them to the conflgurations $f^{3} z^{2}, f^{3} d s$, and $f^{3} d^{2}$. The lowest level of $U \mathrm{~m}$ belongs to the ${ }^{4} \mathrm{I}^{\circ}$ term of the $f^{3} s^{2}$ configuration, from which the ${ }^{\circ} \mathrm{L}^{\circ}$ term of $\mathrm{U}_{\mathrm{I}}$ is derived by adding to it a $d$ electron to give the configuration $f^{3} d s^{2}$. Schuurman's report also confirms the levels 'L:, $5 \mathrm{~K}_{5}^{\circ}$, and ${ }^{7} \mathrm{M}$ and their electron configurations as given in table 2 above. 


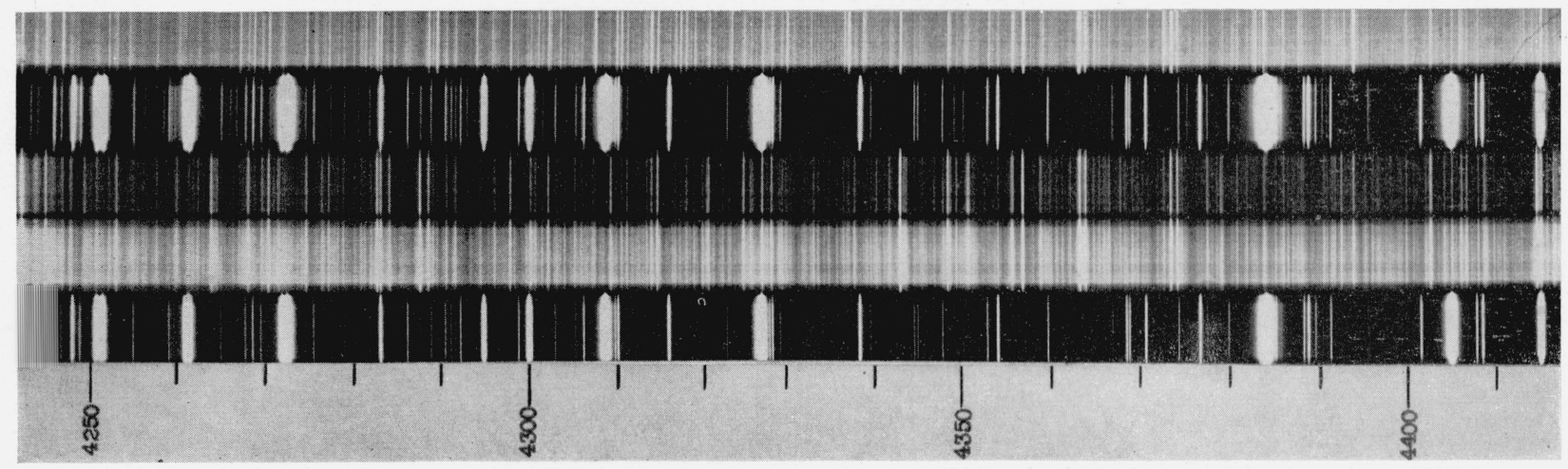

FigURE 1.-Top to bottom: U arc in vacuum; Fe arc in air; U spark in vacuum; U spark in air; Fe arc in air.
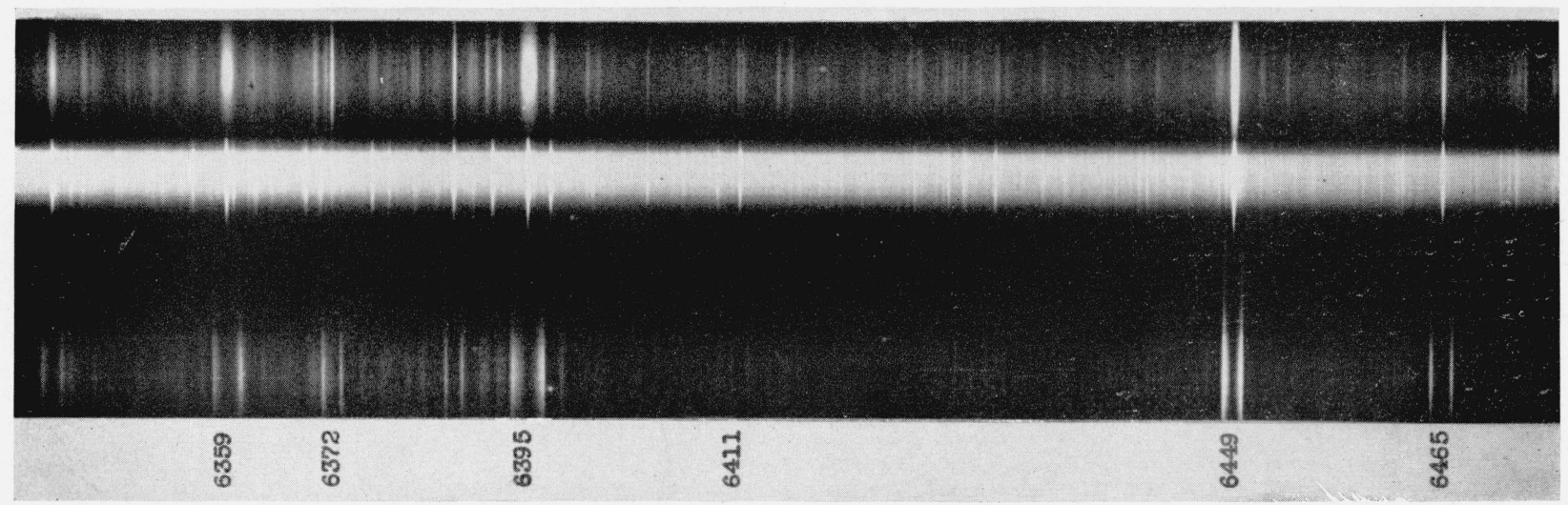

F1GURE 2.-Zeeman effect of uranium; p-components; no-field; n-components. 
The configuration $f^{3} d s^{2}$ yields 158 terms, comprising 386 energy levels distributed among the quintet, triplet, and singlet systems. The $f^{3} d^{2} s$ configuration gives rise to 1,122 terms, with a total of 3,256 levels belonging to the septet, quintet, triplet, and singlet systems. On excitation of the atom, these configurations change into $f^{3} d p s, f^{3} p s^{2}$, and $f^{3} d^{2} p$, depending on which electron is shifted from the low-energy configurations. Each of these excited states yields an enormous number of levels, of even parity, of which more than 275 have now been found. It is obvious why the observed spectrum is so complex.

The even levels of table 3 , in combination with the odd levels of table 2, account for most of the stronger lines of the spectrum, as recorded in table 4. For only a small number of the levels have $g$-values been found, but their inner quantum numbers can be uniquely determined from their combinatory properties. The present state of the analysis does not permit assignment of the even levels to their proper electron configurations. In table 3 the symbol for the even levels is made up of the first three figures of the level value, followed by the inner quantum number as a subscript. This follows the scheme adopted by Laporte and Mack [13] for tungsten.

TABLE 3.-Even levels of U I

\begin{tabular}{|c|c|c|c|}
\hline Term symbol & Term value & Term symbol & Term value \\
\hline $\begin{array}{l}179_{3} \\
185_{3} \\
196_{3} \\
203_{3} \\
219_{3}\end{array}$ & $\begin{array}{l}17968.7 \\
18530.8 \\
19668.4 \\
20391.5 \\
21940.6\end{array}$ & $\begin{array}{l}210_{4} \\
215_{4} \\
220_{4} \\
223_{4} \\
224_{4}\end{array}$ & $\begin{array}{l}21062.3 \\
21545.1 \\
22038.0 \\
22365.0 \\
22383.4\end{array}$ \\
\hline $\begin{array}{l}227_{3} \\
234_{3} \\
251_{3} \\
268_{3} \\
294_{3}\end{array}$ & $\begin{array}{l}\text { 22774. } 1 \\
23464.1 \\
25160.7 \\
26855.4 \\
29413.7\end{array}$ & $\begin{array}{l}225_{4} \\
231_{4} \\
234_{4} \\
235_{4} \\
238_{4}\end{array}$ & $\begin{array}{l}22584.5 \\
23186.9 \\
23430.1 \\
23560.6 \\
23825.4\end{array}$ \\
\hline $\begin{array}{l}295_{3} \\
296_{3} \\
302_{3} \\
340_{3} \\
161_{4}\end{array}$ & $\begin{array}{l}\text { 29430. } 3 \\
29644.6 \\
30222.4 \\
34065.4 \\
16121.9\end{array}$ & $\begin{array}{l}247_{4} \\
249_{4} \\
252_{4} \\
256_{4} \\
265_{4}\end{array}$ & $\begin{array}{l}24757.2 \\
24940.5 \\
25224.1 \\
25653.3 \\
26516.8\end{array}$ \\
\hline $\begin{array}{l}174_{4} \\
178_{4} \\
181_{4} \\
185_{4} \\
191_{4}\end{array}$ & $\begin{array}{l}17468.2 \\
17893.8 \\
18186.0 \\
18526.9 \\
19127.2\end{array}$ & $\begin{array}{l}271_{4} \\
283_{4} \\
284_{4} \\
285_{4} \\
292_{4}\end{array}$ & $\begin{array}{l}27184.1 \\
28430.5 \\
28454.0 \\
28543.4 \\
29250.5\end{array}$ \\
\hline $\begin{array}{l}192_{4} \\
195_{4} \\
197_{4} \\
201_{4} \\
205_{4}\end{array}$ & $\begin{array}{l}\text { 19192. } 4 \\
19552.5 \\
19740.7 \\
20148.0 \\
20569.2\end{array}$ & $\begin{array}{l}297_{4} \\
303_{4} \\
304_{4} \\
309_{4} \\
312_{4}\end{array}$ & $\begin{array}{l}29719.9 \\
30303.7 \\
30499.1 \\
30979.7 \\
31221.3\end{array}$ \\
\hline
\end{tabular}

TABLE 3.-Even levels of $\mathrm{U}_{\mathrm{I}}$-Continued

\begin{tabular}{|c|c|c|c|}
\hline Term symbol & Term value & Term symbol & Term value \\
\hline $\begin{array}{l}313_{4} \\
316_{4} \\
321_{4} \\
116_{5} \\
122_{5}\end{array}$ & $\begin{array}{l}31243.5 \\
31633.9 \\
32141.2 \\
11614.0 \\
12227.8\end{array}$ & $\begin{array}{l}173_{6} \\
182_{6} \\
187_{6} \\
197_{6} \\
202_{6}\end{array}$ & $\begin{array}{l}17361.9 \\
18253.9 \\
18759.2 \\
19783.4 \\
20218.8\end{array}$ \\
\hline $\begin{array}{l}134_{5} \\
157_{5} \\
169_{5} \\
179_{5} \\
184_{5}\end{array}$ & $\begin{array}{l}13463.4 \\
15720.7 \\
16929.8 \\
17908.2 \\
18406.5\end{array}$ & $\begin{array}{l}204_{6} \\
206_{6} \\
209_{6} \\
212_{6} \\
215_{6}\end{array}$ & $\begin{array}{l}20420.5 \\
20661.5 \\
20943.4 \\
21265.1 \\
21584.7\end{array}$ \\
\hline $\begin{array}{l}189_{5} \\
194_{5} \\
196_{5} \\
201_{5} \\
203_{5}\end{array}$ & $\begin{array}{l}18932.8 \\
19471.9 \\
19647.5 \\
20114.3 \\
20311.5\end{array}$ & $\begin{array}{l}217_{6} \\
218_{6} \\
220_{6} \\
224_{6} \\
225_{6}\end{array}$ & $\begin{array}{l}21766.5 \\
21768.0 \\
22056.3 \\
22464.3 \\
22582.7\end{array}$ \\
\hline $\begin{array}{l}206_{5} \\
208_{5} \\
210_{5} \\
213_{5} \\
216_{5}\end{array}$ & $\begin{array}{l}20621.2 \\
20851.6 \\
21078.7 \\
21330.0 \\
21637.0\end{array}$ & $\begin{array}{l}227_{6} \\
228_{6} \\
235_{6} \\
237_{6} \\
240_{6}\end{array}$ & $\begin{array}{l}22754.1 \\
22862.4 \\
23572.0 \\
23715.3 \\
24026.2\end{array}$ \\
\hline $\begin{array}{l}223_{5} \\
233_{5} \\
234_{5} \\
235_{5} \\
239_{5}\end{array}$ & $\begin{array}{l}22377.7 \\
23325.2 \\
23432.8 \\
23486.7 \\
23932.8\end{array}$ & $\begin{array}{l}244_{6} \\
246_{6} \\
252_{6} \\
253_{6} \\
254_{6}\end{array}$ & $\begin{array}{l}24433.2 \\
24671.4 \\
25235.7 \\
25349.0 \\
25462.6\end{array}$ \\
\hline $\begin{array}{l}244_{5} \\
245_{5} \\
249_{5} \\
250_{5} \\
251_{5}\end{array}$ & $\begin{array}{l}24448.0 \\
24535.3 \\
24906.8 \\
25017.1 \\
25178.1\end{array}$ & $\begin{array}{l}257_{6} \\
258_{6} \\
259_{6} \\
261_{6} \\
262_{6}\end{array}$ & $\begin{array}{l}25791.6 \\
25825.6 \\
25938.2 \\
26103.7 \\
26225.5\end{array}$ \\
\hline $\begin{array}{l}252_{5} \\
253_{5} \\
258_{5} \\
263_{5} \\
265_{5}\end{array}$ & $\begin{array}{l}25255.4 \\
25319.2 \\
25805.8 \\
26305.0 \\
26566.9\end{array}$ & $\begin{array}{l}265_{6} \\
266_{6} \\
267_{6} \\
268_{6} \\
269_{6}\end{array}$ & $\begin{array}{l}26550.4 \\
26631.4 \\
26715.5 \\
26758.8 \\
26791.6\end{array}$ \\
\hline $\begin{array}{l}269_{5} \\
273_{5} \\
276_{5} \\
277_{5} \\
278_{5}\end{array}$ & $\begin{array}{l}26920.7 \\
27381.7 \\
27682.2 \\
27778.0 \\
27791.1\end{array}$ & $\begin{array}{l}270_{6} \\
274_{6} \\
275_{6} \\
276_{6} \\
277_{6}\end{array}$ & $\begin{array}{l}27072.4 \\
27499.4 \\
27605.7 \\
27615.8 \\
27744.0\end{array}$ \\
\hline $\begin{array}{l}281_{5} \\
284_{5} \\
285_{5} \\
286_{5} \\
287_{5}\end{array}$ & $\begin{array}{l}28188.3 \\
28444.5 \\
28503.5 \\
28562.7 \\
28650.3\end{array}$ & $\begin{array}{l}278_{6} \\
279_{6} \\
280_{6} \\
281_{6} \\
284_{6}\end{array}$ & $\begin{array}{l}27941.2 \\
28022.9 \\
28053.1 \\
28098.9 \\
28470.2\end{array}$ \\
\hline $\begin{array}{l}292_{5} \\
301_{5} \\
309_{5} \\
310_{5} \\
311_{5}\end{array}$ & $\begin{array}{l}29232.6 \\
30143.1 \\
30936.6 \\
30993.0 \\
31129.5\end{array}$ & $\begin{array}{l}288_{6} \\
289_{6} \\
290_{6} \\
291_{6} \\
292_{6}\end{array}$ & $\begin{array}{l}28860.9 \\
28874.9 \\
29033.6 \\
29109.8 \\
29126.1\end{array}$ \\
\hline $\begin{array}{l}313_{5} \\
314_{5} \\
315_{5} \\
317_{5} \\
319_{5}\end{array}$ & $\begin{array}{l}31339.8 \\
31467.6 \\
31488.2 \\
31744.2 \\
31946.0\end{array}$ & $\begin{array}{l}294_{6} \\
295_{6} \\
298_{6} \\
299_{6} \\
300_{6}\end{array}$ & $\begin{array}{l}29400.9 \\
29558.8 \\
29865.5 \\
29986.4 \\
30294.3\end{array}$ \\
\hline $\begin{array}{l}323_{5} \\
146_{6} \\
156_{6} \\
165_{6} \\
170_{6}\end{array}$ & $\begin{array}{l}32317.8 \\
14643.9 \\
15638.4 \\
16505.8 \\
17070.5\end{array}$ & $\begin{array}{l}301_{6} \\
302_{6} \\
303_{6} \\
304_{6} \\
305_{6}\end{array}$ & $\begin{array}{l}30335.0 \\
30451.4 \\
30490.3 \\
30500.1 \\
30586.7\end{array}$ \\
\hline
\end{tabular}


TABLE 3.-Even levels of U $\mathrm{I}$-Continued

\begin{tabular}{|c|c|c|c|}
\hline Term symbol & Term value & Term symbol & Term value \\
\hline $\begin{array}{l}306_{6} \\
307_{6} \\
308_{6} \\
311_{6} \\
315_{6}\end{array}$ & $\begin{array}{l}30636.7 \\
30875.6 \\
30894.5 \\
31135.0 \\
31551.5\end{array}$ & $\begin{array}{l}318_{7} \\
319_{7} \\
320_{7} \\
321_{7} \\
326_{7}\end{array}$ & $\begin{array}{l}31968.1 \\
31974.4 \\
32016.7 \\
32098.2 \\
32641.8\end{array}$ \\
\hline $\begin{array}{l}324_{6} \\
325_{6} \\
334_{6} \\
336_{6} \\
156_{7}\end{array}$ & $\begin{array}{l}32490.6 \\
32495.7 \\
33412.2 \\
33639.6 \\
15631.9\end{array}$ & $\begin{array}{l}327_{7} \\
335_{7} \\
340_{7} \\
194_{8} \\
205_{8}\end{array}$ & $\begin{array}{l}32774.2 \\
33570.6 \\
34059.9 \\
19489.0 \\
20528.9\end{array}$ \\
\hline $\begin{array}{l}169_{7} \\
197_{7} \\
198_{7} \\
204_{7} \\
207_{7}\end{array}$ & $\begin{array}{l}16900.4 \\
19826.7 \\
19885.4 \\
20464.5 \\
20766.5\end{array}$ & $\begin{array}{l}223_{8} \\
227_{8} \\
231_{8} \\
239_{8} \\
244_{8}\end{array}$ & $\begin{array}{l}22383.1 \\
22789.8 \\
23110.8 \\
23926.7 \\
24451.7\end{array}$ \\
\hline $\begin{array}{l}223_{7} \\
226_{7} \\
229_{7} \\
230_{7} \\
231_{7}\end{array}$ & $\begin{array}{l}22368.4 \\
22633.2 \\
22918.6 \\
23057.7 \\
23197.0\end{array}$ & $\begin{array}{l}245_{8} \\
253_{8} \\
257_{8} \\
259_{8} \\
261_{8}\end{array}$ & $\begin{array}{l}\text { 24581. } 2 \\
25388.9 \\
25789.0 \\
25918.1 \\
26192.4\end{array}$ \\
\hline $\begin{array}{l}235_{7} \\
237_{7} \\
238_{7} \\
240_{7} \\
241_{7}\end{array}$ & $\begin{array}{l}23543.5 \\
23779.2 \\
23848.6 \\
24066.6 \\
24185.8\end{array}$ & $\begin{array}{l}263_{8} \\
264_{8} \\
265_{8} \\
266_{8} \\
269_{8}\end{array}$ & $\begin{array}{l}\text { 26313. } 3 \\
26454.1 \\
26492.1 \\
26597.0 \\
26979.3\end{array}$ \\
\hline $\begin{array}{l}243_{7} \\
245_{7} \\
256_{7} \\
261_{7} \\
262_{7}\end{array}$ & $\begin{array}{l}24333.8 \\
24560.4 \\
25672.5 \\
26208.8 \\
26274.8\end{array}$ & $\begin{array}{l}270_{8} \\
271_{8} \\
272_{8} \\
274_{8} \\
278_{8}\end{array}$ & $\begin{array}{l}26989.1 \\
27086.4 \\
27150.5 \\
27477.6 \\
27818.5\end{array}$ \\
\hline $\begin{array}{l}263_{7} \\
266_{7} \\
273_{7} \\
278_{7} \\
279_{7}\end{array}$ & $\begin{array}{l}\text { 26391. } 3 \\
26608.5 \\
27324.5 \\
27887.0 \\
27965.9\end{array}$ & $\begin{array}{l}289_{8} \\
290_{8} \\
294_{8} \\
296_{8} \\
300_{8}\end{array}$ & $\begin{array}{l}28993.7 \\
29037.2 \\
29413.2 \\
29612.7 \\
30027.2\end{array}$ \\
\hline $\begin{array}{l}280_{7} \\
281_{7} \\
282_{7} \\
283_{7} \\
284_{7}\end{array}$ & $\begin{array}{l}28048.2 \\
28118.8 \\
28152.7 \\
28285.8 \\
28451.1\end{array}$ & $\begin{array}{l}307_{8} \\
309_{8} \\
312_{8} \\
314_{8} \\
319_{8}\end{array}$ & $\begin{array}{l}30737.1 \\
30986.3 \\
31279.1 \\
31445.3 \\
31923.1\end{array}$ \\
\hline $\begin{array}{l}285_{7} \\
287_{7} \\
288_{7} \\
291_{7} \\
297_{7}\end{array}$ & $\begin{array}{l}28566.4 \\
28798.9 \\
28895.6 \\
29107.1 \\
29790.7\end{array}$ & $\begin{array}{l}324_{8} \\
341_{8} \\
238_{9} \\
245_{9} \\
256_{9}\end{array}$ & $\begin{array}{l}32477.8 \\
34105.4 \\
23843.7 \\
24517.3 \\
25626.6\end{array}$ \\
\hline $\begin{array}{l}298_{7} \\
299_{7} \\
300_{7} \\
302_{7} \\
306_{7}\end{array}$ & $\begin{array}{l}29797.2 \\
29837.6 \\
29958.1 \\
30279.1 \\
30642.8\end{array}$ & $\begin{array}{l}279_{9} \\
293_{9} \\
295_{9} \\
312_{9} \\
321_{9}\end{array}$ & $\begin{array}{l}27969.7 \\
29339.3 \\
29550.3 \\
31270.3 \\
32108.4\end{array}$ \\
\hline $\begin{array}{l}310_{7} \\
311_{7} \\
312_{7} \\
313_{7} \\
314_{7}\end{array}$ & $\begin{array}{l}\text { 31024. } 8 \\
31166.2 \\
31276.0 \\
31301.1 \\
31358.6\end{array}$ & & \\
\hline
\end{tabular}

TABLE 4.-Classified lines of $\mathrm{U}_{\mathbf{I}}$

\begin{tabular}{|c|c|c|c|}
\hline$\lambda_{\mathrm{air}} \mathbf{A}$ & Intensity & $\nu_{\mathrm{vac}} \mathrm{Cm}^{-1}$ & Term combination \\
\hline $\begin{array}{l}11384.2 \\
11167.8 \\
10647.6 \\
10555.02 \\
10259.42\end{array}$ & $\begin{array}{r}2 \\
3 \\
3 \\
20 \\
3\end{array}$ & $\begin{array}{l}8781.71 \\
8951.83 \\
9389.23 \\
9471.57 \\
9744.47\end{array}$ & $\begin{array}{r}\mathrm{C}_{7}^{\circ}-169_{7} \\
\mathrm{C}_{7}^{\circ}-170_{6} \\
{ }^{7} \mathrm{M}_{6}^{\circ}-156_{6} \\
{ }^{7} \mathrm{M}_{6}^{\circ}-157_{5} \\
\mathrm{~B}_{7}^{\circ}-170_{6}\end{array}$ \\
\hline $\begin{array}{r}10073.54 \\
9932.76 \\
9917.04 \\
9868.30 \\
9818.86\end{array}$ & $\begin{array}{l}2 \\
3 \\
2 \\
3 \\
5\end{array}$ & $\begin{array}{r}9924.28 \\
10064.94 \\
10080.89 \\
10130.68 \\
10181.69\end{array}$ & $\begin{array}{r}{ }^{7} \mathrm{~K}_{6}^{\circ}-169_{5} \\
{ }^{7} \mathrm{~K}_{6}^{\circ}-1700_{6} \\
\mathrm{~B}_{8}^{\circ}-207_{7}^{7} \\
{ }^{5} \mathrm{H}_{4}^{\circ}-161_{4} \\
\mathrm{D}_{7}^{\circ}-205_{8}\end{array}$ \\
\hline $\begin{array}{l}9653.20 \\
9645.82 \\
9642.00 \\
9595.06 \\
9395.37\end{array}$ & $\begin{array}{l}4 \\
5 \\
2 \\
4 \\
5\end{array}$ & $\begin{array}{l}10356.42 \\
10364.35 \\
10368.45 \\
10419.18 \\
10640.63\end{array}$ & $\begin{array}{r}{ }^{7} \mathrm{~K}_{6}^{\circ}-173_{6} \\
\mathrm{~B}_{3}^{\circ}-174_{4} \\
{ }^{5} \mathrm{~K}_{6}^{\circ}-146_{6} \\
\mathrm{D}_{7}^{\circ}-2077 \\
\mathrm{C}_{7}^{\circ}-187_{6}\end{array}$ \\
\hline $\begin{array}{l}9385.89 \\
9329.43 \\
9305.18 \\
9257.66 \\
9148.48\end{array}$ & $\begin{array}{r}5 \\
6 \\
4 \\
10 \\
4\end{array}$ & $\begin{array}{l}10651.37 \\
10715.83 \\
10743.76 \\
10798.91 \\
10927.78\end{array}$ & $\begin{array}{r}{ }^{7} \mathrm{M}_{6}^{\circ}-169_{7} \\
\mathrm{~A}_{9}^{\circ}-238_{9} \\
{ }^{7} \mathrm{~K}_{5}^{\circ}-165_{6} \\
\mathrm{~A}_{9}^{\circ}-239_{8} \\
\mathrm{~B}_{7}^{\circ}-182_{6}\end{array}$ \\
\hline $\begin{array}{l}9139.57 \\
9093.68 \\
8996.08 \\
8951.94 \\
8887.76\end{array}$ & $\begin{array}{r}10 \\
25 \\
2 \\
20 \\
3\end{array}$ & $\begin{array}{l}10938.44 \\
10993.64 \\
11112.91 \\
11167.70 \\
11248.35\end{array}$ & $\begin{array}{r}{ }^{5} \mathrm{H}_{4}^{\circ}-169_{5} \\
{ }^{5} \mathrm{~K}_{5}^{\circ}-116_{5} \\
{ }^{7} \mathrm{M}_{6}^{\circ}-173_{6} \\
{ }^{7} \mathrm{~K}_{5}^{\circ}-169_{5} \\
{ }^{7} \mathrm{~K}_{6}^{\circ}-182_{6}\end{array}$ \\
\hline $\begin{array}{l}8828.49 \\
8792.34 \\
8777.69 \\
8753.68 \\
8728.75\end{array}$ & $\begin{array}{r}2 \\
3 \\
10 \\
25 \\
8\end{array}$ & $\begin{array}{l}11323.86 \\
11370.42 \\
11389.40 \\
11420.64 \\
11453.25\end{array}$ & $\begin{array}{l}\mathrm{A}_{9}-244_{8} \\
\mathrm{C}_{7}^{\circ}-194_{8} \\
\mathrm{~A}_{9}^{\circ}-245_{9} \\
\mathrm{D}_{7}^{\circ}-218_{6} \\
\mathrm{~A}_{9}^{\circ} 245_{8}\end{array}$ \\
\hline $\begin{array}{l}8710.76 \\
8618.47 \\
8607.96 \\
8574.59 \\
8570.52\end{array}$ & $\begin{array}{r}40 \\
8 \\
600 \\
30 \\
120\end{array}$ & $\begin{array}{l}11476.91 \\
11599.81 \\
11613.97 \\
11659.17 \\
11664.70\end{array}$ & $\begin{array}{r}{ }^{5} \mathrm{H}_{4}-174_{4} \\
{ }^{7} \mathrm{~K}_{5}^{\circ}-173_{6} \\
{ }^{5} \mathrm{~L}_{6}^{\circ}-116_{5} \\
{ }^{7} \mathrm{M}_{6}^{\circ}-179_{5} \\
\mathrm{C}_{7}^{\circ} 197_{6}\end{array}$ \\
\hline $\begin{array}{l}8567.71 \\
8557.32 \\
8540.19 \\
8496.09 \\
8450.02\end{array}$ & $\begin{array}{r}40 \\
30 \\
100 \\
100 \\
400\end{array}$ & $\begin{array}{l}11668.53 \\
11682.70 \\
11706.13 \\
11766.89 \\
11831.04\end{array}$ & $\begin{array}{r}{ }^{5} \mathrm{I}_{4}^{\circ}-161_{4} \\
\mathrm{~B}_{8}^{\circ}-223_{7} \\
{ }^{7} \mathrm{~K}_{5}^{\circ}-174_{4} \\
\mathrm{C}_{7}^{\circ}-198_{7} \\
\mathrm{~A}_{7}^{\circ}-156_{7}\end{array}$ \\
\hline $\begin{array}{l}8445.37 \\
8441.20 \\
8399.25 \\
8389.16 \\
8381.86\end{array}$ & $\begin{array}{r}400 \\
80 \\
15 \\
40 \\
120\end{array}$ & $\begin{array}{l}11837.56 \\
11843.41 \\
11902.56 \\
11916.87 \\
11927.25\end{array}$ & $\begin{array}{r}\mathrm{A}_{7}^{\circ}-156_{6} \\
\mathrm{~A}_{8}^{\circ}-194_{8} \\
{ }^{5} \mathrm{H}_{4}^{\circ}-178_{4} \\
{ }^{5} \mathrm{H}_{4}^{\circ}-179_{5} \\
{ }^{7} \mathrm{~K}_{6}^{\circ}-189_{5}\end{array}$ \\
\hline $\begin{array}{l}8346.75 \\
8316.39 \\
8270.05 \\
8262.05 \\
8259.44\end{array}$ & $\begin{array}{r}80 \\
6 \\
15 \\
150 \\
10\end{array}$ & $\begin{array}{l}11977.43 \\
12021.15 \\
12088.51 \\
12100.21 \\
12104.04\end{array}$ & $\begin{array}{r}{ }^{5} \mathrm{H}_{4}^{\circ}-179_{3} \\
\mathrm{D}_{7}^{\circ}-223_{7} \\
\mathrm{~B}_{3}^{\circ}-192_{4} \\
\mathrm{C}_{7}^{\circ}-202_{6} \\
\mathrm{~B}_{8}^{\circ}-227_{8}\end{array}$ \\
\hline $\begin{array}{l}8240.51 \\
8230.83 \\
8223.09 \\
8175.84 \\
8174.30\end{array}$ & $\begin{array}{r}20 \\
40 \\
200 \\
20 \\
250\end{array}$ & $\begin{array}{l}12131.84 \\
12146.11 \\
12157.54 \\
12227.81 \\
12230.11\end{array}$ & $\begin{array}{r}{ }^{7} \mathrm{~K}_{5}^{\circ}-178_{4} \\
{ }^{7} \mathrm{~K}_{5}^{\circ}-179_{5} \\
{ }^{7} \mathrm{M}_{6}^{\circ}-184_{5} \\
{ }^{5} \mathrm{~L}_{6}^{\circ}-1222_{5} \\
{ }^{5} \mathrm{~K}_{6}^{\circ}-165_{6}\end{array}$ \\
\hline $\begin{array}{l}8153.71 \\
8137.21 \\
8126.59 \\
8055.61 \\
8034.79\end{array}$ & $\begin{array}{l}20 \\
30 \\
15 \\
30 \\
40\end{array}$ & $\begin{array}{l}12260.99 \\
12285.85 \\
12301.91 \\
12410.30 \\
12442.46\end{array}$ & $\begin{array}{l}\mathrm{A}_{9}^{\circ}-253_{8} \\
\mathrm{D}_{7}^{\circ}-226_{7} \\
\mathrm{C}_{7}^{\circ}-204_{\circ} \\
\mathrm{C}_{7}-205_{8} \\
\mathrm{D}_{7}^{\circ}-227_{8}\end{array}$ \\
\hline
\end{tabular}




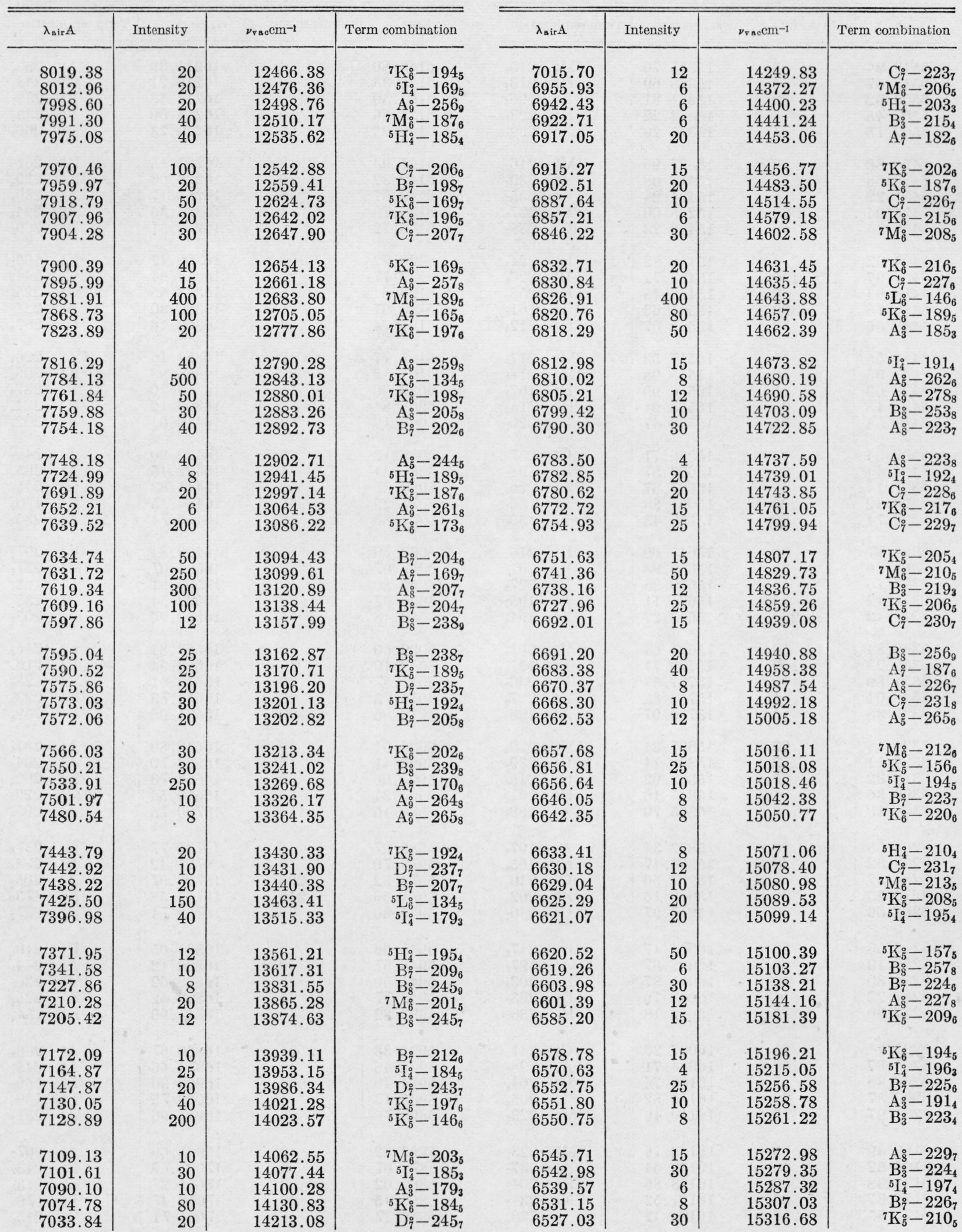




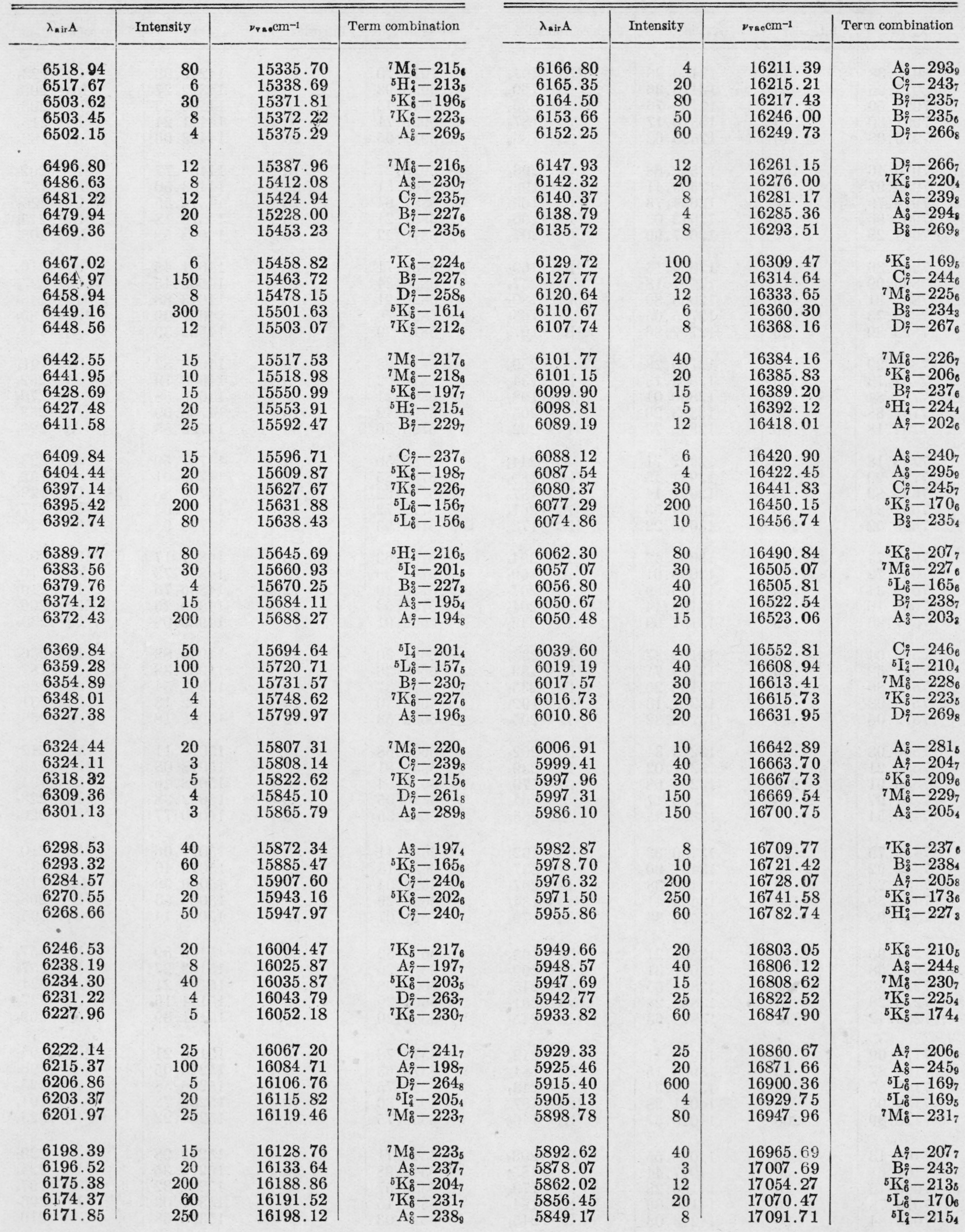




\begin{tabular}{|c|c|c|c|c|c|c|c|}
\hline $\begin{array}{l}5836.03 \\
5835.19 \\
5831.82 \\
5819.01 \\
5817.92\end{array}$ & $\begin{array}{r}80 \\
8 \\
10 \\
8 \\
8\end{array}$ & $\begin{array}{l}17130.19 \\
17132.67 \\
17142.57 \\
17180.30 \\
17183.50\end{array}$ & $\begin{array}{r}D_{7}^{\circ}-274_{8} \\
B_{8}^{\circ}-278_{8} \\
A_{7}^{\circ}-209_{6} \\
{ }^{7} \mathrm{~K}_{6}^{\circ}-241_{7} \\
{ }^{5} \mathrm{I}_{4}^{\circ}-216_{6}\end{array}$ & $\begin{array}{l}5545.75 \\
5534.72 \\
5531.26 \\
5527.98 \\
5526.33\end{array}$ & $\begin{array}{r}4 \\
50 \\
100 \\
80 \\
30\end{array}$ & $\begin{array}{l}18026.83 \\
18062.76 \\
18074.05 \\
18084.80 \\
18090.18\end{array}$ & $\begin{array}{r}\mathrm{A}_{8}^{\circ}-256_{7} \\
\mathrm{~B}_{7}^{\circ}-253_{8} \\
\mathrm{C}_{7}-261_{8} \\
{ }^{7} \mathrm{M}_{8}^{\circ}-243_{7} \\
\mathrm{C}_{7}-261_{7}\end{array}$ \\
\hline $\begin{array}{l}5814.42 \\
5813.83 \\
5802.11 \\
5800.78 \\
5787.60\end{array}$ & $\begin{array}{r}25 \\
20 \\
60 \\
8 \\
6\end{array}$ & $\begin{array}{l}17193.87 \\
17195.61 \\
17230.34 \\
17234.28 \\
17273.55\end{array}$ & $\begin{array}{r}\mathrm{A}_{\mathrm{a}}-210_{4} \\
{ }^{5} \mathrm{H}_{\mathrm{i}}-231_{4} \\
\mathrm{C}_{7}-253_{6} \\
\mathrm{~B}_{7}^{\circ}-245_{7} \\
{ }^{5} \mathrm{~K}_{5}^{\circ}-178_{4}\end{array}$ & $\begin{array}{l}5522.20 \\
5517.16 \\
5511.49 \\
5507.77 \\
5502.18\end{array}$ & $\begin{array}{r}5 \\
10 \\
250 \\
10 \\
60\end{array}$ & $\begin{array}{l}18103.70 \\
18120.25 \\
18138.88 \\
18151.14 \\
18169.57\end{array}$ & $\begin{array}{r}\mathrm{D}_{7}^{\circ}-284_{7} \\
\mathrm{~B}_{3}^{\circ}-252_{4} \\
{ }^{5} \mathrm{~K}_{5}^{\circ}-187_{6} \\
\mathrm{~A}_{9}^{\circ}-312_{8} \\
\mathrm{~A}_{3}^{\circ}-220_{4}\end{array}$ \\
\hline $\begin{array}{l}5784.11 \\
5782.81 \\
5780.59 \\
5771.05 \\
5767.43\end{array}$ & $\begin{array}{r}8 \\
10 \\
100 \\
30 \\
15\end{array}$ & $\begin{array}{l}17283.96 \\
17287.86 \\
17294.49 \\
17323.08 \\
17333.95\end{array}$ & $\begin{array}{r}\mathrm{B}_{8}^{\circ}-279_{0} \\
{ }^{5} \mathrm{~K}_{5}^{\circ}-179_{6} \\
{ }^{7} \mathrm{M}_{6}^{\circ}-235_{7} \\
{ }^{7} \mathrm{M}_{8}-235_{6} \\
{ }^{5} \mathrm{H}_{4}^{\circ}-233_{5}\end{array}$ & $\begin{array}{l}5501.82 \\
5500.69 \\
5497.75 \\
5496.43 \\
5494.59\end{array}$ & $\begin{array}{r}25 \\
120 \\
15 \\
150 \\
25\end{array}$ & $\begin{array}{l}18170.78 \\
18174.51 \\
18184.23 \\
18188.59 \\
18194.68\end{array}$ & $\begin{array}{r}{ }^{7} \mathrm{~K}_{5}^{\circ}-239_{5} \\
\mathrm{~A}_{5}^{\circ}-297_{4} \\
{ }^{7} \mathrm{M}_{6}^{\circ}-244_{6} \\
{ }^{5} \mathrm{~K}_{6}^{\circ}-224_{6} \\
\mathrm{C}_{7}^{\circ}-263_{8}\end{array}$ \\
\hline $\begin{array}{l}5732.74 \\
5731.87 \\
5731.49 \\
5716.87 \\
5715.69\end{array}$ & $\begin{array}{r}5 \\
10 \\
8 \\
20 \\
10\end{array}$ & $\begin{array}{l}17438.86 \\
17441.49 \\
17442.64 \\
17487.25 \\
17490.86\end{array}$ & $\begin{array}{r}{ }^{5} \mathrm{H}_{4}-234_{4} \\
{ }^{5} \mathrm{H}_{4}-234_{5} \\
{ }^{7} \mathrm{~K}_{6}-244_{5} \\
{ }^{5} \mathrm{I}_{4}-219_{3} \\
{ }^{5} \mathrm{~K}_{6}-217_{8}\end{array}$ & $\begin{array}{l}5452.41 \\
5445.87 \\
5441.09 \\
5431.35 \\
5426.68\end{array}$ & $\begin{array}{r}40 \\
10 \\
6 \\
50 \\
20\end{array}$ & $\begin{array}{l}18335.44 \\
18357.44 \\
18373.59 \\
18406.54 \\
18422.38\end{array}$ & $\begin{array}{r}\mathrm{C}_{7}-264_{8} \\
\mathrm{~K}_{6}^{\circ}-226_{7} \\
\mathrm{C}_{7}^{\circ}-265_{8} \\
{ }^{5} \mathrm{~L}_{6}^{\circ}-184_{5} \\
{ }^{7} \mathrm{M}_{6}^{\circ}-246_{6}\end{array}$ \\
\hline $\begin{array}{l}5714.20 \\
5702.85 \\
5699.87 \\
5695.18 \\
5694.85\end{array}$ & $\begin{array}{r}6 \\
10 \\
20 \\
6 \\
4\end{array}$ & $\begin{array}{l}17495.41 \\
17530.24 \\
17539.41 \\
17553.85 \\
17554.88\end{array}$ & $\begin{array}{r}{ }^{5} \mathrm{H}_{4}-235_{5} \\
{ }^{7} \mathrm{M}_{6}^{\circ}-237_{7} \\
\mathrm{D}_{7}-278_{7} \\
\mathrm{C}_{7}-256_{7} \\
{ }^{7} \mathrm{~K}_{6}^{\circ}-245_{7}\end{array}$ & $\begin{array}{l}5418.10 \\
5414.76 \\
5410.24 \\
5406.87 \\
5404.90\end{array}$ & $\begin{array}{r}6 \\
30 \\
80 \\
100 \\
8\end{array}$ & $\begin{array}{l}18451.53 \\
18462.92 \\
18478.34 \\
18489.86 \\
18496.60\end{array}$ & $\begin{array}{r}\mathrm{D}_{7}^{\circ}-287_{7} \\
\mathrm{~B}_{7}^{\circ}-257_{8} \\
5 \mathrm{~K}_{6}^{\circ}-227_{6} \\
\mathrm{C}_{7}-266_{7} \\
\mathrm{~A}_{3}^{\circ}-223_{4}\end{array}$ \\
\hline $\begin{array}{l}5691.35 \\
5690.18 \\
5685.19 \\
5680.37 \\
5674.25\end{array}$ & $\begin{array}{r}15 \\
3 \\
12 \\
8 \\
8\end{array}$ & $\begin{array}{l}17565.65 \\
17569.29 \\
17584.68 \\
17599.61 \\
17618.60\end{array}$ & $\begin{array}{r}{ }^{5} \mathrm{~K}_{5}^{\circ}-181_{4} \\
{ }^{5} \mathrm{H}_{4}^{\circ}-235_{4} \\
{ }^{5} \mathrm{I}_{4}^{\circ}-220_{4} \\
{ }^{7} \mathrm{M}_{6}^{\circ}-238_{7} \\
\mathrm{D}_{7}^{\circ}-279_{7}\end{array}$ & $\begin{array}{l}5401.90 \\
5399.54 \\
5390.28 \\
5389.46 \\
5385.54\end{array}$ & $\begin{array}{r}30 \\
10 \\
10 \\
6 \\
100\end{array}$ & $\begin{array}{l}18506.87 \\
18514.98 \\
18546.77 \\
18549.60 \\
18563.10\end{array}$ & $\begin{array}{r}{ }^{5} \mathrm{~K}_{5}^{\circ}-191_{4} \\
\mathrm{~A}_{3}-224_{4} \\
\mathrm{~A}_{8}-261_{8} \\
\mathrm{~B}_{3}^{\circ}-256_{4} \\
\mathrm{~A}_{8}^{\circ}-261_{7}\end{array}$ \\
\hline $\begin{array}{l}5669.42 \\
5658.26 \\
5646.49 \\
5645.92 \\
5640.30\end{array}$ & $\begin{array}{r}25 \\
15 \\
4 \\
40 \\
40\end{array}$ & $\begin{array}{l}17633.60 \\
17668.39 \\
17705.23 \\
17706.99 \\
17724.63\end{array}$ & $\begin{array}{r}{ }^{5} \mathrm{~K}_{5}-182_{6} \\
{ }^{7} \mathrm{~K}_{5}^{\circ}-234_{4} \\
\mathrm{~A}_{5}^{\circ}-292_{4} \\
\mathrm{C}_{7}^{\circ}-258_{6} \\
{ }^{7} \mathrm{~K}_{5}^{\circ}-235_{5}\end{array}$ & $\begin{array}{l}5384.22 \\
5382.94 \\
5379.96 \\
5378.68 \\
5377.16\end{array}$ & $\begin{array}{r}50 \\
100 \\
6 \\
6 \\
12\end{array}$ & $\begin{array}{l}18567.64 \\
18572.07 \\
18582.34 \\
18586.78 \\
18592.03\end{array}$ & $\begin{array}{r}\mathrm{A}_{7}^{\circ}-223_{7} \\
{ }^{5} \mathrm{~K}_{5}^{\circ}-192_{4} \\
\mathrm{~A}_{7}^{\circ}-223_{8} \\
5 \mathrm{~K}_{6}^{\circ}-228_{6} \\
\mathrm{~B}_{7}^{\circ}-259_{8}\end{array}$ \\
\hline $\begin{array}{l}5634.38 \\
5625.43 \\
5622.54 \\
5621.51 \\
5620.78\end{array}$ & $\begin{array}{r}80 \\
5 \\
30 \\
100 \\
200\end{array}$ & $\begin{array}{l}17743.26 \\
17771.49 \\
17780.63 \\
17783.89 \\
17786.21\end{array}$ & $\begin{array}{r}\mathrm{A}_{8}^{\circ}-253_{8} \\
\mathrm{D}_{7}^{\circ}-281_{7} \\
{ }^{5} \mathrm{~K}_{6}^{\circ}-220_{6} \\
\mathrm{~A}_{7}^{\circ}-215_{6} \\
{ }^{5} \mathrm{~K}_{5}^{\circ}-184_{5}\end{array}$ & $\begin{array}{l}5375.76 \\
5375.50 \\
5371.35 \\
5359.43 \\
5356.58\end{array}$ & $\begin{array}{l}80 \\
40 \\
15 \\
20 \\
20\end{array}$ & $\begin{array}{l}18596.86 \\
18597.76 \\
18612.13 \\
18653.54 \\
18663.46\end{array}$ & $\begin{array}{l}\mathrm{C}_{7}^{\circ}-267_{6} \\
\mathrm{~A}_{5}^{\circ}-301_{5} \\
\mathrm{~B}_{7}^{\circ}-259_{6} \\
\mathrm{~B}_{8}^{\circ}-293_{9} \\
\mathrm{~A}_{7}^{\circ}-224_{6}\end{array}$ \\
\hline $\begin{array}{l}5616.88 \\
5616.58 \\
5614.74 \\
5613.26 \\
5610.89\end{array}$ & $\begin{array}{r}10 \\
80 \\
40 \\
30 \\
150\end{array}$ & $\begin{array}{l}17798.53 \\
17799.51 \\
17805.34 \\
17810.04 \\
17817.56\end{array}$ & $\begin{array}{r}{ }^{7} \mathrm{~K}_{5}^{\circ}-235_{4} \\
\mathrm{C}_{7}-259_{8} \\
\mathrm{D}_{7}^{\circ}-282_{7} \\
{ }^{7} \mathrm{~K}_{5}^{\circ}-235_{6} \\
{ }^{7} \mathrm{M}_{6}^{\circ}-240_{7}\end{array}$ & $\begin{array}{l}5355.58 \\
5355.37 \\
5354.36 \\
5348.99 \\
5341.50\end{array}$ & $\begin{array}{r}30 \\
15 \\
12 \\
12 \\
120\end{array}$ & $\begin{array}{l}18666.92 \\
18667.66 \\
18671.18 \\
18689.95 \\
18716.14\end{array}$ & $\begin{array}{r}7 \mathrm{~K}_{6}^{\circ}-256_{7} \\
\mathrm{~A}_{8}^{\circ}-263_{8} \\
{ }^{7} \mathrm{~K}_{5}^{\circ}-244_{6} \\
\mathrm{D}_{7}^{\circ}-290_{8} \\
\mathrm{~A}_{3}-225_{4}\end{array}$ \\
\hline $\begin{array}{l}5591.10 \\
5584.62 \\
5577.46 \\
5573.59 \\
5573.07\end{array}$ & $\begin{array}{r}30 \\
40 \\
8 \\
60 \\
60\end{array}$ & $\begin{array}{l}17880.61 \\
17901.36 \\
17924.34 \\
17936.80 \\
17938.47\end{array}$ & $\begin{array}{r}\mathrm{B}_{8}^{\circ}-285_{7} \\
{ }^{7} \mathrm{~K}_{8}^{\circ}-249_{5} \\
{ }^{5} \mathrm{I}_{4}^{\circ}-223_{5} \\
{ }^{7} \mathrm{M}_{6}^{\circ}-241_{7} \\
\mathrm{D}_{7}^{\circ}-283_{7}\end{array}$ & $\begin{array}{l}5338.26 \\
5336.54 \\
5329.26 \\
5327.32 \\
5324.03\end{array}$ & $\begin{array}{r}10 \\
120 \\
150 \\
40 \\
20\end{array}$ & $\begin{array}{l}18727.51 \\
18733.55 \\
18759.14 \\
18765.96 \\
18777.57\end{array}$ & $\begin{array}{r}\mathrm{B}_{8}^{\circ}-294_{8} \\
{ }^{5} \mathrm{I}_{4}^{\circ}-231_{4} \\
{ }^{5} \mathrm{~L}_{6}^{\circ}-187_{6} \\
{ }^{5} \mathrm{H}_{4}^{\circ}-2477_{4} \\
\mathrm{~B}_{7}^{\circ}-261_{6}\end{array}$ \\
\hline $\begin{array}{l}5572.09 \\
5568.48 \\
5564.17 \\
5559.88 \\
5546.97\end{array}$ & $\begin{array}{r}15 \\
40 \\
300 \\
20 \\
6\end{array}$ & $\begin{array}{l}17941.63 \\
17953.24 \\
17967.16 \\
17981.03 \\
18022.87\end{array}$ & $\begin{array}{r}{ }^{5} \mathrm{H}_{i}^{\circ}-239_{5} \\
{ }^{7} \mathrm{~K}_{5}^{\circ}-237_{6} \\
\mathrm{~A}_{7}^{\circ}-218_{6} \\
\mathrm{~A}_{8}-256_{9} \\
\mathrm{~B}_{7}^{\circ}-253_{6}\end{array}$ & $\begin{array}{l}5322.78 \\
5319.01 \\
5315.27 \\
5308.54 \\
5304.57\end{array}$ & $\begin{array}{r}60 \\
15 \\
150 \\
300 \\
40\end{array}$ & $\begin{array}{l}18781.98 \\
18795.28 \\
18808.51 \\
18832.34 \\
18846.44\end{array}$ & $\begin{array}{r}{ }^{5} \mathrm{~K}_{8}-230_{7} \\
\mathrm{~A}_{9}^{\circ}-312_{8} \\
\mathrm{~A}_{8}-264_{8} \\
\mathrm{~A}_{7}^{\circ}-226_{7} \\
\mathrm{~A}_{8}^{\circ}-265_{8}\end{array}$ \\
\hline
\end{tabular}


TABLE 4.-Classified lines of $\mathrm{U} \mathrm{I}-$ Continued

\begin{tabular}{|c|c|c|c|}
\hline$\lambda_{\mathrm{air}} \mathrm{A}$ & Intensity & $\nu_{\mathrm{Vac}} \mathrm{Cm}^{-1}$ & Term combination \\
\hline $\begin{array}{l}5303.09 \\
5300.57 \\
5299.44 \\
5297.44 \\
5286.93\end{array}$ & $\begin{array}{r}20 \\
120 \\
100 \\
150 \\
60\end{array}$ & $\begin{array}{l}18851.70 \\
18860.68 \\
18864.69 \\
18871.81 \\
18909.30\end{array}$ & $\begin{array}{r}{ }^{5} \mathrm{~K}_{5}^{\circ}-194_{5} \\
\mathrm{C}_{7}-269_{8} \\
\mathrm{~B}_{8}^{\circ}-295_{9} \\
{ }^{5} \mathrm{I}_{4}^{\circ}-233_{5} \\
{ }^{7} \mathrm{~K}_{5}^{\circ}-246_{6}\end{array}$ \\
\hline $\begin{array}{l}5283.58 \\
5281.99 \\
5280.54 \\
5280.38 \\
5275.93\end{array}$ & $\begin{array}{r}50 \\
15 \\
50 \\
300 \\
80\end{array}$ & $\begin{array}{l}18921.32 \\
18927.00 \\
18932.22 \\
18932.78 \\
18948.76\end{array}$ & $\begin{array}{r}5 \mathrm{~K}_{6}^{\circ}-231_{7} \\
\mathrm{~B}_{8}^{\circ}-296_{8} \\
{ }^{5} \mathrm{~K}_{5}^{\circ}-195_{4} \\
{ }^{5} \mathrm{~L}_{6}^{\circ}-189_{5} \\
\mathrm{~B}_{7}^{\circ}-262_{7}\end{array}$ \\
\hline $\begin{array}{l}5275.19 \\
5274.68 \\
5272.01 \\
5270.62 \\
5267.42\end{array}$ & $\begin{array}{r}12 \\
10 \\
100 \\
100 \\
30\end{array}$ & $\begin{array}{l}18951.40 \\
18953.24 \\
18962.85 \\
18967.82 \\
18979.37\end{array}$ & $\begin{array}{c}\mathrm{A}_{8}^{\circ}-266_{8} \\
\mathrm{~A}_{7}-227_{6} \\
\mathrm{~A}_{8}-266_{7} \\
\mathrm{C}_{7}-271_{8} \\
{ }^{5} \mathrm{I}_{4}^{\circ}-234_{5}\end{array}$ \\
\hline $\begin{array}{l}5267.10 \\
5265.38 \\
5265.25 \\
5264.76 \\
5259.90\end{array}$ & $\begin{array}{r}5 \\
50 \\
60 \\
8 \\
80\end{array}$ & $\begin{array}{l}18980.50 \\
18986.72 \\
18987.18 \\
18988.94 \\
19006.48\end{array}$ & $\begin{array}{r}\mathrm{A}_{9}^{\circ}-321_{9} \\
{ }^{7} \mathrm{M}_{6}^{\circ}-252_{6} \\
\mathrm{~B}_{7}^{\circ}-263_{8} \\
\mathrm{~A}_{7}^{\circ}-227_{8} \\
{ }^{7} \mathrm{M}_{6}^{\circ}-252_{5}\end{array}$ \\
\hline $\begin{array}{l}5258.73 \\
5254.13 \\
5244.70 \\
5242.33 \\
5234.16\end{array}$ & $\begin{array}{r}4 \\
4 \\
20 \\
12 \\
120\end{array}$ & $\begin{array}{l}19010.73 \\
19027.37 \\
19061.56 \\
19070.19 \\
19099.95\end{array}$ & $\begin{array}{r}{ }^{5} \mathrm{I}_{4}^{\circ}-234_{3} \\
{ }^{5} \mathrm{~K}_{5}^{\circ}-196_{5} \\
\mathrm{~A}_{7}^{\circ}-228_{6} \\
{ }^{7} \mathrm{M}_{6}^{\circ}-253_{5} \\
{ }^{7} \mathrm{M}_{6}^{\circ}-253_{6}\end{array}$ \\
\hline $\begin{array}{l}5232.81 \\
5232.18 \\
5231.02 \\
5229.30 \\
5226.51\end{array}$ & $\begin{array}{l}30 \\
10 \\
20 \\
25 \\
15\end{array}$ & $\begin{array}{l}19104.88 \\
19107.18 \\
19111.43 \\
19117.69 \\
19127.93\end{array}$ & $\begin{array}{l}\mathrm{B}_{8}^{\circ}-297_{7} \\
5 \mathrm{I}_{4}^{\circ}-235_{4} \\
\mathrm{~B}_{8}^{\circ}-298_{9} \\
\mathrm{~A}_{7}^{\circ}-229_{7} \\
\mathrm{~B}_{7}^{\circ}-264_{8}\end{array}$ \\
\hline $\begin{array}{l}5221.90 \\
5218.56 \\
5216.92 \\
5215.18 \\
5212.74\end{array}$ & $\begin{array}{l}80 \\
12 \\
80 \\
40 \\
10\end{array}$ & $\begin{array}{l}19144.82 \\
19157.06 \\
19163.06 \\
19169.46 \\
19178.46\end{array}$ & $\begin{array}{l}{ }^{7} \mathrm{~K}_{5}^{\circ}-249_{5} \\
{ }^{5} \mathrm{~K}_{6}^{\circ}-234_{5} \\
{ }^{5} \mathrm{~K}_{5}^{\circ}-197_{6} \\
{ }^{5} \mathrm{H}_{4}^{\circ}-251_{3} \\
{ }^{7} \mathrm{~K}_{5}^{\circ}-249_{4}\end{array}$ \\
\hline $\begin{array}{l}5210.48 \\
5203.20 \\
5201.47 \\
5198.00 \\
5192.01\end{array}$ & $\begin{array}{r}8 \\
10 \\
20 \\
12 \\
25\end{array}$ & $\begin{array}{l}19186.76 \\
19213.62 \\
19220.01 \\
19232.83 \\
19255.03\end{array}$ & $\begin{array}{r}{ }^{5} \mathrm{H}_{4}^{\circ}-251_{5} \\
{ }^{7} \mathrm{M}_{6}^{\circ}-254_{6} \\
{ }^{7} \mathrm{~K}_{6}^{\circ}-262_{6} \\
{ }^{5} \mathrm{H}_{4}^{\circ}-252_{4} \\
{ }^{7} \mathrm{~K}_{5}^{\circ}-250_{5}\end{array}$ \\
\hline $\begin{array}{l}5191.51 \\
5189.54 \\
5189.21 \\
5188.57 \\
5180.89\end{array}$ & $\begin{array}{r}40 \\
6 \\
30 \\
25 \\
50\end{array}$ & $\begin{array}{l}19256.88 \\
19264.20 \\
19265.40 \\
19267.78 \\
19296.35\end{array}$ & $\begin{array}{r}\mathrm{A}_{7}^{\circ}-230_{7} \\
{ }^{5} \mathrm{H}_{4}^{\circ}-252_{5} \\
\mathrm{D}_{7}^{\circ}-296_{8} \\
{ }^{5} \mathrm{~K}_{6}^{\circ}-235_{7} \\
{ }^{5} \mathrm{~K}_{6}^{\circ}-235_{6}\end{array}$ \\
\hline $\begin{array}{l}5180.03 \\
5177.22 \\
5170.90 \\
5168.79 \\
5168.25\end{array}$ & $\begin{array}{r}12 \\
8 \\
12 \\
20 \\
10\end{array}$ & $\begin{array}{l}19299.53 \\
19310.04 \\
19333.63 \\
19341.50 \\
19343.52\end{array}$ & $\begin{array}{r}{ }^{7} \mathrm{~K}_{6}^{\circ}-263_{5} \\
\mathrm{~A}_{7}^{\circ}-231_{8} \\
\mathrm{~A}_{8}^{\circ}-269_{8} \\
\mathrm{~B}_{8}^{\circ}-300_{8} \\
\mathrm{~A}_{8}-270_{8}\end{array}$ \\
\hline $\begin{array}{l}5166.74 \\
5164.14 \\
5160.67 \\
5156.03 \\
5155.50\end{array}$ & $\begin{array}{r}20 \\
200 \\
15 \\
25 \\
8\end{array}$ & $\begin{array}{l}19349.17 \\
19358.93 \\
19371.96 \\
19389.37 \\
19391.37\end{array}$ & $\begin{array}{l}\mathrm{A}_{5}^{\circ}-308_{6} \\
\mathrm{C}_{7}-274_{8} \\
{ }^{5} \mathrm{I}_{4}^{\circ}-238_{4} \\
\mathrm{~B}_{7}^{\circ}-267_{6} \\
\mathrm{~A}_{5}^{\circ}-309_{5}\end{array}$ \\
\hline $\begin{array}{l}5154.22 \\
5148.95 \\
5146.98 \\
5144.52 \\
5144.11\end{array}$ & $\begin{array}{l}15 \\
15 \\
40 \\
60 \\
10\end{array}$ & $\begin{array}{l}19396.20 \\
19416.04 \\
19423.46 \\
19432.78 \\
19434.31\end{array}$ & $\begin{array}{r}\mathrm{A}_{7}^{\circ}-231_{7} \\
\mathrm{~K}_{5}^{\circ}-251_{5} \\
{ }^{7} \mathrm{M}_{6}^{\circ}-256_{7} \\
\mathrm{~B}_{7}^{\circ}-268_{6} \\
\mathrm{~A}_{5}^{\circ}-309_{4}\end{array}$ \\
\hline
\end{tabular}

TABLE 4.-Classified lines of $\mathrm{U} \mathrm{I}$-Continued

\begin{tabular}{|c|c|c|c|}
\hline$\lambda_{\mathrm{sir}} \mathrm{A}$ & Intensity & $\nu_{\mathrm{VAC}} \mathrm{Cm}^{-1}$ & Term combination \\
\hline $\begin{array}{l}5142.72 \\
5142.40 \\
5139.98 \\
5136.74 \\
5134.19\end{array}$ & $\begin{array}{r}10 \\
60 \\
35 \\
6 \\
8\end{array}$ & $\begin{array}{l}19439.56 \\
19440.78 \\
19449.94 \\
19462.18 \\
19471.85\end{array}$ & $\begin{array}{r}{ }^{5} \mathrm{~K}_{6}^{\circ}-237_{6} \\
\mathrm{~A}_{8}^{\circ}-271_{8} \\
\mathrm{D}_{7}^{\circ}-298_{7} \\
{ }^{7} \mathrm{~K}_{5}^{\circ}-252_{4} \\
{ }^{5} \mathrm{~L}_{6}^{\circ}-194_{5}\end{array}$ \\
\hline $\begin{array}{l}5132.20 \\
5130.16 \\
5129.34 \\
5128.36 \\
5125.86\end{array}$ & $\begin{array}{r}40 \\
8 \\
15 \\
15 \\
12\end{array}$ & $\begin{array}{l}19479.42 \\
19487.14 \\
19490.28 \\
19493.98 \\
19503.51\end{array}$ & $\begin{array}{r}{ }^{5} \mathrm{I}_{4}^{\circ}-239_{5} \\
\mathrm{C}_{7}-275_{6} \\
\mathrm{D}_{7}^{\circ}-299_{7} \\
{ }^{5} \mathrm{~K}_{5}^{\circ}-201_{5} \\
{ }^{5} \mathrm{~K}_{6}^{\circ}-237_{7}\end{array}$ \\
\hline $\begin{array}{l}5125.49 \\
5119.51 \\
5110.69 \\
5107.68 \\
5106.74\end{array}$ & $\begin{array}{l}25 \\
25 \\
30 \\
40 \\
30\end{array}$ & $\begin{array}{l}19504.89 \\
19527.71 \\
19561.39 \\
19572.94 \\
19576.54\end{array}$ & $\begin{array}{r}\mathrm{A}_{8}^{\circ}-272_{8} \\
{ }^{5} \mathrm{~K}_{5}^{\circ}-201_{4} \\
{ }^{7} \mathrm{~K}_{6}^{\circ}-265_{5} \\
{ }^{5} \mathrm{~K}_{6}^{\circ}-238_{7} \\
{ }^{7} \mathrm{M}_{6}^{\circ}-258_{6}\end{array}$ \\
\hline $\begin{array}{l}5104.03 \\
5101.74 \\
5101.01 \\
5097.88 \\
5093.89\end{array}$ & $\begin{array}{r}8 \\
10 \\
40 \\
6 \\
20\end{array}$ & $\begin{array}{l}19586.93 \\
19595.71 \\
19598.51 \\
19610.54 \\
19625.91\end{array}$ & $\begin{array}{r}{ }^{7} \mathrm{~K}_{5}^{\circ}-253_{6} \\
\mathrm{~A}_{3}^{\circ}-234_{3} \\
{ }^{5} \mathrm{~K}_{5}^{\circ}-202_{6} \\
\mathrm{D}_{7}^{\circ}-300_{7} \\
{ }^{7} \mathrm{~K}_{6}^{\circ}-266_{6}\end{array}$ \\
\hline $\begin{array}{l}5088.29 \\
5086.83 \\
5084.52 \\
5080.99 \\
5076.99\end{array}$ & $\begin{array}{r}120 \\
15 \\
10 \\
3 \\
10\end{array}$ & $\begin{array}{l}19647.52 \\
19653.15 \\
19662.06 \\
19675.75 \\
19691.23\end{array}$ & $\begin{array}{r}{ }^{5} \mathrm{~L}_{6}^{\circ}-196_{5} \\
\mathrm{~B}_{7}^{\circ}-269_{8} \\
{ }^{5} \mathrm{H}_{4}^{\circ}-256_{4} \\
\mathrm{~A}_{5}^{\circ}-312_{4} \\
{ }^{5} \mathrm{~K}_{5}^{\circ}-203_{5}^{\circ}\end{array}$ \\
\hline
\end{tabular}

$\mathrm{A}_{3}^{\circ}-235_{4}$

$\mathrm{C}_{7}-278$

$\mathrm{A}_{7}^{\circ}-235_{7}$

${ }^{5} \mathrm{~K}_{6}^{\circ}-240_{6}$

$\mathrm{B}_{3}^{\circ}-268_{3}$

19751.48

19753.34

${ }^{7} \mathrm{~K}_{6}^{\circ}-268_{6}$

$\mathrm{B} \div-271_{8}$

$\mathrm{C}_{7}^{\circ}-278_{7}$

$\mathrm{A}_{7}^{\circ}-235_{6}$

19768.35

19771.18

5056.46

5053.35

19783.35

5051.43

5049.06

5045.39

5042.31

19790.88

19800.18

19814.56

19826.66

19854.65

${ }^{5} \mathrm{~L}_{6}^{\circ}-197_{6}$

${ }^{5} \mathrm{~K}_{6}^{\circ}-240_{7}$

${ }^{5} \mathrm{~K}_{5}^{\circ}-204_{6}$

${ }^{5} \mathrm{H}_{4}-2588_{5}$

${ }^{5} \mathrm{~L}_{6}^{\circ}-197_{7}$

${ }^{7} \mathrm{M}_{6}^{\circ}-261_{6}$

5027.38

5022.64

5021.18

5019.89

5016.25

5012.92

5011.42

5009.39

5008.68

5004.01

19885.54

19904.30

19910.09

19915.20

19929.66

${ }^{5} \mathrm{~L}_{6}^{\circ}-198_{7}$

$\mathrm{C}_{7}-279_{6}$

${ }^{5} \mathrm{~K}_{6}^{\circ}-241_{7}$

$7 \mathrm{~K}_{6}^{\circ}-269_{5}$
$\mathrm{C}_{7}^{\circ}-280_{7}$

19942.90

19948.90

19956.94

19959.79

19978.42

$\mathrm{A}_{5}^{\circ}-315_{5}$

$5 \mathrm{~K}_{5}-205_{4}$

$\mathrm{A}_{3}^{\circ}-238_{4}$

${ }^{7} \mathrm{M}_{6}^{\circ}-261_{7}$

$\mathrm{A}_{7}^{\circ}-237$ ?

4999.95

4998.56

4998.41

4990.12

4988.32

4986.69

4985.82

4984.62

4984.12

4981.95
19994.66

20000.20

20000.78

20034.04

20041. 24

20047.82

20051.32

20056. 14

20058. 12

20066.88
${ }^{5} \mathrm{I}_{4}-244_{5}$

$\mathrm{C}_{7}^{\circ}-281_{7}$

${ }^{5} \mathrm{~K}_{5}^{\circ}-206_{5}$

$\mathrm{C}_{7}-282_{7}$

${ }^{5} \mathrm{~K}_{5}^{\circ}-206_{6}$

$\mathrm{A}_{7}-238_{7}$

$\mathrm{B}_{8}^{\circ}-307_{8}$

${ }^{7} \mathrm{M}_{6}^{\circ}-263_{5}$

${ }^{5} \mathrm{~K}_{6}^{\circ}-243_{7}$
${ }^{7} \mathrm{~K}_{6}^{\circ}-270_{8}$ 


\begin{tabular}{|c|c|c|c|c|c|c|c|}
\hline$\lambda_{\mathrm{a} \text { ir } \mathrm{A}}$ & Intensity & $\nu_{\mathrm{Vac}} \mathrm{cm}^{-1}$ & Term combination & $\lambda_{\mathrm{air}} \mathrm{A}$ & Intensity & $\nu_{\mathrm{vac}} \mathrm{cm}^{-1}$ & Term combination \\
\hline 4978.22 & 8 & 20081.91 & ${ }^{5} \mathrm{I}_{4}^{\circ}-245_{5}$ & 4782.81 & 8 & 20902.38 & ${ }^{5} \mathrm{~K}_{6}^{\circ}-251_{5}$ \\
\hline 4972.72 & 10 & 20104.12 & $\mathrm{D}_{7}^{\circ}-302_{6}$ & 4779.09 & 8 & 20918.66 & $\mathrm{C}_{7}-290_{8}$ \\
\hline 4970.20 & 20 & 20114.32 & ${ }^{5} \mathrm{~L}_{6}^{\circ}-201_{5}$ & 4777.67 & 80 & 20924.86 & ${ }^{5} \mathrm{~K}_{5}^{\circ}-215_{4}^{\circ}$ \\
\hline 4967.33 & 120 & 20125.93 & $\mathrm{~A}_{7}-239_{8}$ & 4773.43 & 60 & 20943.45 & ${ }^{5} \mathrm{~L}_{6}^{\circ}-209_{6}$ \\
\hline 4963.31 & & 20142.26 & ${ }^{7} \mathrm{M}_{6}^{\circ}-263_{7}$ & 4768.66 & 60 & 20964.39 & ${ }^{5} \mathrm{~K}_{5}^{\circ}-215_{6}$ \\
\hline 4961.10 & 20 & 20151.20 & $\mathrm{~B}_{7}^{\circ}-274_{8}$ & 4761.34 & 15 & 20996.65 & ${ }^{7} \mathrm{~K}_{5}^{\circ}-268_{0}$ \\
\hline 4959.55 & 25 & 20157.53 & ${ }^{5} \mathrm{~K}_{6}^{\circ}-244_{6}$ & 4756.81 & 120 & 21016.64 & ${ }^{5} \mathrm{~K}_{5}^{\circ}-216_{5}$ \\
\hline 4955.90 & 10 & 20172.34 & ${ }^{5} \mathrm{~K}_{6}^{\circ}-244_{5}$ & 4750.72 & 8 & 21043.56 & ${ }^{5} \mathrm{~K}_{6}^{\circ}-253_{5}$ \\
\hline 4955.78 & 120 & 20172.83 & $\mathrm{~A}_{8}^{\circ}-278_{8}$ & 4744.30 & 12 & 21072.04 & $\mathrm{~A}_{3}^{\circ}-249_{4}$ \\
\hline 4954.96 & 50 & 20176.18 & ${ }^{7} \mathrm{~K}_{5}^{\circ}-259_{6}$ & 4744.04 & 12 & 21073.22 & ${ }^{5} K_{6}^{\circ}-253_{6}$ \\
\hline 4949.41 & 5 & 20198.80 & $\mathrm{~A}_{5}^{\circ}-317_{5}$ & 4743.53 & 50 & 21075.48 & ${ }^{7} \mathrm{M}_{6}^{\circ}-273_{7}$ \\
\hline 4944.52 & 80 & 20218.80 & ${ }^{5} \mathrm{~L}_{6}^{\circ}-202_{6}$ & 4739.50 & 4 & 21093.40 & ${ }^{7} \mathrm{~K}_{6}^{\circ}-281_{6}$ \\
\hline 4941.47 & 10 & 20231.25 & ${ }^{5} \mathrm{~K}_{5}^{\circ}-208_{5}$ & 4738.44 & 30 & 21098.10 & $\mathrm{D}_{7}-314_{8}$ \\
\hline 4934.56 & 10 & 20259.60 & ${ }^{5} \mathrm{~K}_{6}^{\circ}-245_{5}$ & 4732.43 & 5 & 21124.92 & $\mathrm{~B} ;-284$ \\
\hline 4933.06 & 50 & 20265.75 & $\mathrm{~A}_{7}^{\circ}-240_{7}$ & 4730.68 & 25 & 21132.70 & ${ }^{7} \mathrm{M}_{6}-273_{5}$ \\
\hline 4928.44 & 100 & 20284.74 & ${ }^{5} \mathrm{~K}_{6}^{\circ}-245_{7}$ & 4727.66 & 20 & 21146.21 & ${ }^{5} \mathrm{~K}_{5}^{\circ}-217_{6}$ \\
\hline 4921.38 & 30 & 20313.84 & ${ }^{5} \mathrm{H}_{4}^{0}-263_{5}$ & 4727.34 & 25 & 21147.63 & ${ }^{5} \mathrm{~K}_{5}^{0}-218_{6}$ \\
\hline 4919.13 & 25 & 20323.13 & ${ }^{5} \mathrm{~K}_{5}^{\circ}-209_{6}$ & 4715.68 & 120 & 21199.93 & ${ }^{5} \mathrm{I}_{4}^{\circ}-256_{4}$ \\
\hline 4918.90 & 15 & 20324.07 & $\mathrm{~A}_{8}-279_{9}$ & 4706.74 & 8 & 21240.18 & $\mathrm{~B}_{7}^{\circ}-285$ \\
\hline 4916.88 & 40 & 20332.45 & $\mathrm{C}_{7}-284_{7}$ & 4701.23 & 50 & 21265.10 & ${ }^{5} \mathrm{~L}_{6}^{\circ}-21$ \\
\hline 4910.35 & 120 & 20359.49 & ${ }^{7} \mathrm{M}_{6}^{\circ}-266_{7}$ & 4697.87 & 8 & 21280.32 & ${ }^{7} \mathrm{~K}_{6}-283_{7}$ \\
\hline 4906.29 & 10 & 20376.33 & ${ }^{7} \mathrm{~K}_{6}^{\circ}-273_{5}$ & 4696.06 & 12 & 21288.53 & $\mathrm{~B}_{8}^{\circ}-319_{7}$ \\
\hline 4904.21 & 15 & 20384.98 & $\mathrm{~A}_{7}^{\circ}-241_{7}$ & 4695.23 & 25 & 21292.27 & $\mathrm{~A}_{3}-251_{3}$ \\
\hline 4901.64 & 8 & 20395.68 & ${ }^{5} \mathrm{~K}_{6}^{\circ}-246_{6}$ & 4694.71 & 15 & 21294.64 & $\mathrm{C}_{7}-29$ \\
\hline 4896.39 & 5 & 20417.55 & $\mathrm{~B}_{7}^{\circ}-277_{6}$ & 4691.26 & 10 & 21310.30 & ${ }^{7} \mathrm{~K}_{5}^{\circ}-270_{6}$ \\
\hline 4895.68 & 15 & 20420.51 & ${ }^{5} \mathrm{~L}_{6}-204_{6}$ & 4686.92 & 30 & 21330.00 & ${ }^{5} \mathrm{~L}_{6} \mathrm{O}-213_{5}$ \\
\hline 4889.16 & 8 & 20447.74 & $\mathrm{C}_{7}^{\circ}-285_{7}$ & 4682.00 & 40 & 21352.43 & ${ }^{5} \mathrm{I}_{4}-258_{5}$ \\
\hline 4885.15 & 120 & 20464.51 & ${ }^{5} \mathrm{~L}_{6}-2047$ & 4681.06 & 6 & 21356.74 & ${ }^{7} \mathrm{M}_{6}^{\circ}-275_{6}$ \\
\hline 4879.76 & 10 & 20487.10 & ${ }^{5} \mathrm{I}_{4}^{\circ}-249_{4}$ & 4666.66 & 8 & 21422.65 & $\mathrm{~B}_{8}^{\circ}-321_{0}$ \\
\hline 4878.50 & 30 & 20492.39 & $\mathrm{~B}_{7}-278_{8}$ & 4663.75 & 120 & 21435.98 & ${ }^{5} K_{5}^{\circ}-22$ \\
\hline 4878.16 & 5 & 20493.84 & ${ }^{7} \mathrm{~K}_{6}^{\circ}-274_{6}$ & 4662.83 & 10 & 21440.22 & $\mathrm{C}_{7}-295_{6}$ \\
\hline 4874.35 & 20 & 20509.83 & ${ }^{7} \mathrm{M}_{6}^{\circ}-268_{6}$ & 4661.65 & 30 & 21445.64 & ${ }^{7} \mathrm{~K}_{6}^{\circ}-284_{7}$ \\
\hline 4868.87 & 25 & 20532.95 & $\mathrm{~A}_{7}^{\circ}-243_{7}$ & 4651.13 & 12 & 21494.14 & $\mathrm{C}_{7}-296_{8}$ \\
\hline 4866.59 & 6 & 20542.56 & ${ }^{7} \mathrm{M}_{6}^{\circ}-269_{6}$ & 4643.61 & 50 & 21528.98 & ${ }^{7} \mathrm{M}_{6}^{\circ}-277$ \\
\hline 4866.48 & 4 & 20543.00 & ${ }^{7} \mathrm{~K}_{5}^{\circ}-263_{5}$ & 4639.36 & 6 & 21548.69 & $\mathrm{~B}_{7}^{\circ}-28$ \\
\hline 4856.65 & 50 & 20584.57 & $\mathrm{~B}_{8}-312_{9}$ & 4633.51 & 8 & 21575.90 & $\mathrm{D}_{7}^{\circ}-31$ \\
\hline 4852.96 & 20 & 20600.26 & ${ }^{7} \mathrm{~K}_{8}^{\circ}-275_{6}$ & 4631.62 & 100 & 21584.67 & ${ }^{5} \mathrm{~L}_{6}^{\circ}-215_{6}^{\circ}$ \\
\hline 4850.59 & 8 & 20610.30 & ${ }^{7} \mathrm{~K}_{6}^{\circ}-276_{6}$ & 4630.90 & 8 & 21588.03 & $\mathrm{~A}_{7}^{\circ}-253_{8}$ \\
\hline 4848.00 & 30 & 20621.33 & ${ }^{5} \mathrm{~L}_{6}^{\circ}-206_{5}$ & 4623.91 & 5 & 21620.69 & $\mathrm{D}_{7}^{\circ}-318$ \\
\hline 4845.67 & 15 & 20631.23 & ${ }^{5} \mathrm{~K}_{6}^{0}-249_{5}$ & 4620.44 & 60 & 21636.94 & ${ }^{5} \mathrm{~L}_{6}^{\circ}-21$ \\
\hline 4842.48 & 80 & 20644.82 & ${ }^{5} \mathrm{~K}_{5}^{\circ}-212_{0}$ & 4620.23 & 300 & 21637.88 & ${ }^{7} \mathrm{M}_{6}^{\circ}-278_{7}$ \\
\hline 4841.04 & 12 & 20650.97 & $\mathrm{~A}_{7}^{\circ}-244_{8}$ & 4618.75 & 8 & 21644.85 & ${ }^{7} \mathrm{~K}_{6}^{\circ}-287$ \\
\hline 4835.91 & 8 & 20672.88 & $\mathrm{~B}_{8}^{\circ}-314_{7}$ & 4612.93 & 8 & 21672.16 & $\mathrm{C}_{7}-297$ \\
\hline 4834.18 & 12 & 20680.27 & $\mathrm{C}_{7}-287_{7}$ & 4608.35 & 6 & 21693.66 & $A_{8}^{\circ}-293_{9}$ \\
\hline 4827.86 & 4 & 20707.34 & ${ }^{5} \mathrm{I}_{4}-251_{3}$ & 4605.42 & 4 & 21707.50 & $\mathrm{~B}_{7}^{\circ}-290_{6}$ \\
\hline 4816.84 & 4 & 20754.72 & ${ }^{7} \mathrm{~K}_{5}^{\circ}-265_{4}$ & 4603.43 & 12 & 21716.87 & ${ }^{7} \mathrm{M}_{6}^{\circ}-279_{7}$ \\
\hline 4816.47 & 5 & 20756.30 & $\mathrm{C}_{7}-289_{0}$ & 4599.07 & 4 & 21737.46 & ${ }^{7} \mathrm{~K}_{5}^{\circ}-27$ \\
\hline 4815.71 & 80 & 20759.57 & $\mathrm{~B}_{8}-314_{8}$ & 4594.85 & 8 & 21757.44 & ${ }^{5} \mathrm{~K}_{5}^{\circ}-22$ \\
\hline 4814.11 & 12 & 20766.50 & ${ }^{5} \mathrm{~L}_{6}^{\circ}-207_{7}$ & 4593.65 & 5 & 21763.12 & ${ }^{5} \mathrm{~K}_{5}^{\circ}-22$ \\
\hline 4812.72 & 10 & 20772.47 & ${ }^{7} \mathrm{~K}_{6}^{0}-277_{5}$ & 4592.93 & 15 & 21766.53 & ${ }^{5} \mathrm{~L}_{6}^{0}-21$ \\
\hline 4810.90 & 100 & 20780.36 & $\mathrm{~A}_{7}-245_{8}$ & 4592.63 & 5 & 21767.95 & ${ }^{5} \mathrm{~L}_{6} \mathrm{O}-218$ \\
\hline 4808.03 & 5 & 20792.73 & $\mathrm{~B}_{7}-281_{7}$ & 4588.65 & 15 & 21786.83 & ${ }^{5} \mathrm{H}_{4}^{\circ}-27$ \\
\hline 4805.22 & 3 & 20804.88 & ${ }^{7} \mathrm{~K}_{5}^{\circ}-265_{5}$ & 4577.59 & 4 & 21839.47 & $\mathrm{C}_{7}-30$ \\
\hline 4800.23 & 10 & 20826.54 & $\mathrm{~B}_{7}^{\circ}-282_{7}$ & 4576.64 & 80 & 21843.97 & ${ }^{5} \mathrm{~K}_{5}^{\circ}-224$ \\
\hline 4794.50 & 10 & 20851.44 & ${ }^{5} \mathrm{~L}_{6}-208_{5}$ & 4575.03 & 8 & 21851.68 & ${ }^{5} \mathrm{I}_{4}^{\circ}-26$ \\
\hline 4791.57 & 6 & 20864.18 & ${ }^{5} \mathrm{H}_{4}-268_{3}$ & 4574.27 & 6 & 21855.32 & ${ }^{7} \mathrm{~K}_{6}^{\circ}-28$ \\
\hline 4790.38 & 5 & 20869.36 & $7 \mathrm{~K}_{5}^{\circ}-266_{0}$ & 4571.68 & 8 & 21867.69 & $\mathrm{C}_{7}-29$ \\
\hline 4790.06 & 80 & 20870.73 & $\mathrm{~A}_{7}^{\circ}-246_{6}$ & 4571.24 & 10 & 21869.81 & ${ }^{7} \mathrm{M}_{6}^{\circ}-281_{7}$ \\
\hline 4789.03 & 6 & 20875.22 & $\mathrm{C}_{7}-289_{8}$ & 4563.95 & 40 & 21904.72 & $\mathrm{~A}:-295$ \\
\hline 4787.60 & 8 & 20881.49 & ${ }^{7} \mathrm{~K}_{6}^{\circ}-278_{7}$ & 4558.05 & 25 & 21933.09 & ${ }^{5} \mathrm{~K}_{6}^{\circ}-26$ \\
\hline
\end{tabular}


TABLE 4.-Classified lines of U I-Continued

\begin{tabular}{|c|c|c|c|}
\hline$\lambda_{\mathbf{s i r}} \mathrm{A}$ & Intensity & $\nu_{\text {raecm}}-1$ & Term combination \\
\hline 4554.59 & 4 & 21949.73 & ${ }^{5} \mathrm{~K}_{6}-262_{6}$ \\
\hline 4551.98 & 80 & 21962.31 & ${ }^{5} \mathrm{~K}_{5}-225_{6}$ \\
\hline 4551.58 & 15 & 21964.27 & ${ }^{5} \mathrm{~K}_{5}^{\circ}-225_{4}$ \\
\hline 4550.98 & 12 & 21967.15 & $A_{8}-296_{8}$ \\
\hline 4546.62 & 8 & 21988.24 & $A_{7}-257_{8}$ \\
\hline 4546.10 & 6 & 21990.76 & $A_{i}-257_{s}$ \\
\hline 4544.36 & 15 & 21999.17 & ${ }^{5} \mathrm{~K}_{6}-2627$ \\
\hline 4539.08 & 12 . & 22024.75 & $A_{7}-258_{6}$ \\
\hline 4536.60 & 20 & 22036.76 & ${ }^{9} \mathrm{M}_{6}-283_{7}$ \\
\hline 4532.58 & 12 & 22056.31 & ${ }^{5} \mathrm{~L}_{\S}-22 \mathrm{O}_{6}$ \\
\hline 4531.14 & 10 & 22063.33 & ${ }^{5} I_{4}-265_{4}$ \\
\hline 4526.25 & 6 & 22087.18 & $\mathrm{~B}_{7}-294_{8}$ \\
\hline 4525.98 & 8 & 22088.51 & $\mathrm{~B}_{8}^{\circ}-327_{7}$ \\
\hline 4517.40 & 3 & 22130.42 & $\mathrm{D}_{7}-324_{8}$ \\
\hline 4516.73 & 80 & 22133.72 & $\mathrm{~K}_{\mathrm{j}}-227$ \\
\hline 4514.11 & 10 & 22146.56 & $\mathrm{~B} ;-292_{4}$ \\
\hline 4504.87 & 6 & 22192.01 & $\mathrm{~A}_{8}-2997$ \\
\hline 4504.16 & 5 & 22195.50 & ${ }^{7} \mathrm{M}_{6}-284_{5}$ \\
\hline 4499.92 & 5 & 22216.39 & $\mathrm{C}_{q}-301_{6}$ \\
\hline 4494.71 & 60 & 22242.18 & $\mathrm{~K}_{5}-228$ \\
\hline 4484.81 & 5 & 22291.27 & ${ }^{5} \mathrm{~K}_{6}: 265_{5}$ \\
\hline 4481.07 & 10 & 22309.87 & $\mathrm{~B}_{3}-294_{3}$ \\
\hline 4476.46 & 40 & 22332.82 & ${ }^{5} K_{6}^{\circ}-266_{7}$ \\
\hline 4469.32 & 100 & 22368.51 & ${ }^{3} \mathrm{~L}_{6}-223_{7}$ \\
\hline 4468.70 & 4 & 22371.60 & $\mathrm{C}_{7}-303_{3}$ \\
\hline 4466.71 & 4 & 22381.59 & $\mathrm{C}_{7}-304_{6}$ \\
\hline 4461.44 & 8 & 22408.00 & $\mathrm{~A}_{7}-261_{7}$ \\
\hline 4458.10 & 5 & 22424.79 & $\mathrm{~A}_{7}-262$ \\
\hline 4455.23 & 5 & 22439.23 & ${ }^{5} \mathrm{H}_{4}-283_{4}$ \\
\hline 4449.66 & 5 & 22467.34 & ${ }^{5} \mathrm{I}_{i}-269_{5}$ \\
\hline 4449.52 & 8 & 22468.07 & $\mathrm{C}_{7}-305_{6}$ \\
\hline 4448.90 & 8 & 22471.18 & $\mathrm{~B}_{7}^{\circ}-298_{7}$ \\
\hline 4448.33 & 40 & 22474.05 & $\mathrm{~A}_{7}-262_{7}$ \\
\hline 4446.53 & 12 & 22483.18 & ${ }^{5} \mathrm{~K}_{6}-268_{6}$ \\
\hline 4440.74 & 60 & 22512.49 & $\mathrm{~A}_{7}-263_{8}$ \\
\hline 4438.43 & 6 & 22524.17 & $\mathrm{C}_{7}-306_{7}$ \\
\hline 4435.16 & 6 & 22540.77 & $\mathrm{~B}_{3}-296_{3}$ \\
\hline 4430.08 & 30 & 22566.64 & ${ }^{5} \mathrm{~K}_{5}^{\circ}-231_{4}$ \\
\hline 4426.94 & 80 & 22582.67 & ${ }^{5} \mathrm{~L}_{8}-225_{6}$ \\
\hline 4425.41 & 12 & 22590.47 & $A_{7}-263_{7}$ \\
\hline 4421.22 & 3 & 22611.84 & ${ }^{7} \mathrm{M}_{6} \mathrm{-}-288_{6}$ \\
\hline 4420.40 & 20 & 22616.05 & $\mathrm{~B}_{3}-297_{4}$ \\
\hline 4418.47 & 25 & 22625.93 & ${ }^{7} \mathrm{M}_{6}-289_{6}$ \\
\hline 4414.75 & 8 & 22645.01 & ${ }^{5} \mathrm{~K}_{8}-269_{5}$ \\
\hline 4414.08 & 8 & 22648.46 & $A_{3}-265_{4}$ \\
\hline 4413.14 & 15 & 22653.29 & $\mathrm{~A}_{7}-264_{8}$ \\
\hline 4412.03 & 2 & 22658.98 & ${ }^{5} \mathrm{H}_{4}^{\circ}-287_{5}$ \\
\hline 4405.74 & 20 & 22691.34 & $\mathrm{~A}_{7}-265_{8}$ \\
\hline 4403.07 & 4 & 22705.09 & ${ }^{5} \mathrm{~K}_{5}-233_{5}$ \\
\hline 4398.10 & 4 & 22730.72 & ${ }^{5} Y_{4}-271_{4}$ \\
\hline 4394.45 & 3 & 22749.59 & $A_{7}-265_{6}$ \\
\hline 4393.60 & 200 & 22754.01 & ${ }^{5} \mathrm{~L}_{6}-227_{6}$ \\
\hline 4387.70 & 6 & 22784.61 & ${ }^{7} \mathrm{M}_{6}-290_{6}$ \\
\hline 4385.48 & 6 & 22796.16 & $\mathrm{~A}_{7}-266_{8}$ \\
\hline 4385.38 & 10 & 22796.66 & ${ }^{5} \mathrm{~K}_{6}-270_{6}$ \\
\hline 4383.27 & 50 & 22807.62 & $A_{7}-266_{7}$ \\
\hline 4382.84 & 8 & 22809.86 & ${ }^{5} \mathrm{~K}_{5}-234_{4}$ \\
\hline 4382.34 & 40 & 22812.47 & ${ }^{5} \mathrm{~K}_{5}^{\circ}-234_{5}$ \\
\hline 4378.90 & 3 & 22830.40 & $\mathrm{~A}_{7}-266_{8}$ \\
\hline 4373.60 & 5 & 22858.08 & ${ }^{7} \mathrm{M}_{6}-291_{7}$ \\
\hline
\end{tabular}

Table 4.-Classified lines of U I-Continued

\begin{tabular}{|c|c|c|c|}
\hline$\lambda_{\text {air } A}$ & Intensity & $\mathrm{p}_{\mathrm{rae}} \mathrm{Cm}^{-1}$ & Term combination \\
\hline $\begin{array}{l}4373.08 \\
4372.76 \\
4372.01 \\
4371.76 \\
4369.96\end{array}$ & $\begin{array}{r}4 \\
80 \\
10 \\
100 \\
20\end{array}$ & $\begin{array}{l}22860.80 \\
22862.44 \\
22866.36 \\
22867.66 \\
22877.11\end{array}$ & $\begin{array}{r}{ }^{7} \mathrm{M}_{8}-291_{6} \\
{ }^{5} \mathrm{~L}_{8}-228_{6} \\
{ }^{6} \mathrm{~K}_{5}-235_{5} \\
\mathrm{C}_{7}-309_{8} \\
{ }^{7} \mathrm{M}_{8}-292_{6}\end{array}$ \\
\hline $\begin{array}{l}4368.47 \\
4362.80 \\
4362.05 \\
4357.92 \\
4355.75\end{array}$ & $\begin{array}{r}6 \\
20 \\
300 \\
25 \\
80\end{array}$ & $\begin{array}{l}22884.88 \\
22914.64 \\
22918.58 \\
22940.30 \\
22951.74\end{array}$ & $\begin{array}{r}\mathrm{B}_{8}-335_{7} \\
\mathrm{~A}_{7}-267_{6} \\
{ }^{5} \mathrm{~L}_{6}-2299_{7} \\
{ }^{5} \mathrm{~K}_{\xi}-235_{4} \\
{ }^{5} \mathrm{~A}_{5}^{\circ}-235_{6}\end{array}$ \\
\hline $\begin{array}{l}4352.59 \\
4349.71 \\
4349.07 \\
4343.53 \\
4337.63\end{array}$ & $\begin{array}{r}4 \\
4 \\
5 \\
3 \\
15\end{array}$ & $\begin{array}{l}22968.37 \\
22983.62 \\
22986.97 \\
23016.30 \\
23047.61\end{array}$ & $\begin{array}{r}\mathrm{B} ;-300_{6} \\
{ }^{7} \mathrm{M}_{6}-292^{3} \\
\mathrm{~A}_{\ddagger}-268_{8} \\
\mathrm{C}_{7}-311_{6} \\
\mathrm{C}_{7}-311_{7}\end{array}$ \\
\hline $\begin{array}{l}4337.40 \\
4335.73 \\
4329.42 \\
4328.73 \\
4328.01\end{array}$ & $\begin{array}{r}25 \\
60 \\
6 \\
60 \\
5\end{array}$ & $\begin{array}{l}23048.83 \\
23057.71 \\
23091.31 \\
23095.00 \\
23098.82\end{array}$ & $\begin{array}{r}{ }^{5} \mathrm{~K}_{8}-273_{7} \\
{ }^{6} \mathrm{~L}_{8}-2307 \\
\mathrm{~A}_{8}-307_{7} \\
{ }^{6} \mathrm{~K}_{\S}^{\circ}-237_{6} \\
{ }^{7} \mathrm{~K}_{\sharp}^{\circ}-288_{6}\end{array}$ \\
\hline $\begin{array}{l}4325.38 \\
4323.05 \\
4318.09 \\
4317.07 \\
4316.48\end{array}$ & $\begin{array}{r}4 \\
3 \\
15 \\
30 \\
50\end{array}$ & $\begin{array}{l}23112.88 \\
23125.35 \\
23151.89 \\
23157.37 \\
23160.53\end{array}$ & $\begin{array}{r}{ }^{7} \mathrm{~K}_{5}^{\circ}-289_{6} \\
\mathrm{~B}_{7}^{\circ}-302_{6} \\
{ }^{7} \mathrm{M}_{8}^{\circ}-294_{6} \\
\mathrm{C}_{7}^{\circ}-312_{7} \\
\mathrm{C}_{7}^{\circ}-312_{8}\end{array}$ \\
\hline $\begin{array}{l}4315.82 \\
4313.13 \\
4312.39 \\
4311.31 \\
4309.68\end{array}$ & $\begin{array}{r}5 \\
100 \\
8 \\
4 \\
25\end{array}$ & $\begin{array}{l}23164.09 \\
23178.50 \\
23182.50 \\
23188.29 \\
23197.06\end{array}$ & $\begin{array}{r}\mathrm{B}_{7}^{\circ}-303_{6} \\
\mathrm{~A}_{7}-269_{8} \\
\mathrm{C}_{7}^{\circ}-313_{7} \\
\mathrm{~A}_{7}-270_{8} \\
{ }^{5} \mathrm{~L}_{6}^{\circ}-231_{7}\end{array}$ \\
\hline $\begin{array}{l}4309.17 \\
4308.20 \\
4304.81 \\
4303.80 \\
4301.72\end{array}$ & $\begin{array}{r}30 \\
8 \\
4 \\
6 \\
15\end{array}$ & $\begin{array}{l}23199.82 \\
23205.06 \\
23223.32 \\
23228.79 \\
23240.02\end{array}$ & $\begin{array}{r}\mathrm{B}_{3}^{\circ}-303_{4} \\
{ }^{5} \mathrm{~K}_{5}^{\circ}-238_{4} \\
\mathrm{D}_{7}^{\circ}-335_{7} \\
{ }^{5} \mathrm{I}_{4}^{\circ}-276_{5} \\
\mathrm{C}_{7}^{\circ}-314_{7}\end{array}$ \\
\hline $\begin{array}{l}4297.92 \\
4295.88 \\
4293.30 \\
4288.84 \\
4288.70\end{array}$ & $\begin{array}{r}10 \\
5 \\
30 \\
200 \\
10\end{array}$ & $\begin{array}{l}23260.56 \\
23271.59 \\
23285.59 \\
23309.80 \\
23310.54\end{array}$ & $\begin{array}{r}\mathrm{B}_{7}^{\circ}-305_{6} \\
\mathrm{~A}_{7}^{\circ}-270_{6} \\
0^{7}-271_{8} \\
{ }^{7} \mathrm{M}_{6}^{\circ}-295_{6} \\
\mathrm{~B}_{7}^{\circ}-306_{6}\end{array}$ \\
\hline $\begin{array}{l}4287.75 \\
4287.56 \\
4286.00 \\
4285.74 \\
4285.23\end{array}$ & $\begin{array}{r}6 \\
10 \\
6 \\
8 \\
20\end{array}$ & $\begin{array}{l}23315.70 \\
23316.76 \\
23325.22 \\
23326.67 \\
23329.44\end{array}$ & $\begin{array}{r}\mathrm{A}_{3}^{\circ}-271_{4} \\
\mathrm{~B}_{7}^{\circ}-306_{7} \\
{ }^{5} \mathrm{~L}_{6}^{\circ}-233_{5} \\
\mathrm{C}_{7}^{\circ}-314_{8} \\
{ }^{7} \mathrm{~K}_{6}^{\circ}-301_{6}\end{array}$ \\
\hline $\begin{array}{l}4283.17 \\
4276.10 \\
4273.19 \\
4271.24 \\
4271.12\end{array}$ & $\begin{array}{r}3 \\
5 \\
6 \\
15 \\
3\end{array}$ & $\begin{array}{l}23340.63 \\
23379.25 \\
23395.17 \\
23405.85 \\
23406.50\end{array}$ & $\begin{array}{r}\mathrm{A}_{8}^{\circ}-309_{8} \\
\mathrm{~A}_{8}^{\circ}-310_{7} \\
\mathrm{~B}_{3}^{\circ}-304_{4} \\
{ }^{5} \mathrm{~K}_{5}^{\circ}-240_{6} \\
{ }^{5} \mathrm{~K}_{6}^{\circ}-276_{5}\end{array}$ \\
\hline $\begin{array}{l}4270.25 \\
4266.32 \\
4265.18 \\
4259.88 \\
4259.46\end{array}$ & $\begin{array}{r}4 \\
40 \\
6 \\
6 \\
12\end{array}$ & $\begin{array}{l}23411.27 \\
23432.82 \\
23439.08 \\
23468.23 \\
23470.56\end{array}$ & $\begin{array}{r}\mathrm{B}_{7}^{\circ}-307_{8} \\
{ }^{5} \mathrm{~L}_{6}^{\circ}-234_{5} \\
{ }^{5} \mathrm{H}_{4}^{\circ}-295_{3} \\
{ }^{5} \mathrm{~K}_{6}^{\circ}-277_{6} \\
{ }^{7} \mathrm{~K}_{5}^{\circ}-292_{5}\end{array}$ \\
\hline $\begin{array}{l}4256.91 \\
4256.54 \\
4255.10 \\
4253.71 \\
4249.83\end{array}$ & $\begin{array}{r}10 \\
3 \\
5 \\
8 \\
4\end{array}$ & $\begin{array}{l}23484.64 \\
23486.69 \\
23494.62 \\
23502.29 \\
23523.74\end{array}$ & $\begin{array}{r}{ }^{7} \mathrm{~K}_{6}^{\circ}-303_{6} \\
{ }^{5} \mathrm{~L}_{6}^{\circ}-235_{5} \\
{ }^{7} \mathrm{~K}_{6}^{\circ}-304_{6} \\
{ }^{5} \mathrm{~K}_{6}^{\circ}-277_{5} \\
{ }_{7}^{\circ}-273_{7}\end{array}$ \\
\hline
\end{tabular}


TABLE 4.-Classified lines of U I-Continued

\begin{tabular}{|c|c|c|c|c|c|c|c|}
\hline$\lambda_{\mathrm{air}} \mathrm{A}$ & Intensity & $\nu_{\mathrm{va}_{0}} \mathrm{~cm}^{-1}$ & Term combination & $\lambda_{\mathrm{air}} \mathrm{A}$ & Intensity & $\nu_{\mathrm{VaO}} \mathrm{Cm}^{-1}$ & Term combination \\
\hline $\begin{array}{l}4246.58 \\
4246.26 \\
4241.11 \\
4233.13 \\
4231.67\end{array}$ & $\begin{array}{r}2 \\
100 \\
15 \\
25 \\
60\end{array}$ & $\begin{array}{l}23541.73 \\
23543.52 \\
23572.12 \\
23616.55 \\
23624.71\end{array}$ & $\begin{array}{r}{ }^{7} \mathrm{M}_{6}-297_{7} \\
{ }^{5} \mathrm{~L}_{6}^{\circ}-235_{7} \\
{ }^{5} \mathrm{~L}_{6}-235_{6} \\
{ }^{7} \mathrm{M}_{6}-298_{6} \\
\mathrm{~A}_{8}^{\circ}-312_{9}\end{array}$ & $\begin{array}{l}4123.00 \\
4119.09 \\
4117.91 \\
4116.35 \\
4115.65\end{array}$ & $\begin{array}{l}5 \\
4 \\
4 \\
6 \\
8\end{array}$ & $\begin{array}{l}24247.38 \\
24270.41 \\
24277.34 \\
24286.54 \\
24290.67\end{array}$ & $\begin{array}{r}\mathrm{A}_{7}^{\circ}-280_{7} \\
{ }^{7} \mathrm{~K}_{6}^{\circ}-312_{7} \\
\mathrm{~A}_{8}-319_{8} \\
{ }^{5} \mathrm{~K}_{\mathrm{b}}^{\circ}-249_{5} \\
{ }^{5} \mathrm{~K}_{6}^{\circ}-285_{7}\end{array}$ \\
\hline $\begin{array}{l}4230.51 \\
4226.54 \\
4226.17 \\
4225.32 \\
4224.36\end{array}$ & $\begin{array}{r}6 \\
3 \\
3 \\
3 \\
10\end{array}$ & $\begin{array}{l}23631.18 \\
23653.37 \\
23655.46 \\
23660.21 \\
23665.57\end{array}$ & $\begin{array}{r}{ }^{7} \mathrm{~K}_{6}^{\circ}-306_{6} \\
{ }^{5} \mathrm{H}_{4}^{\circ}-296_{3} \\
\mathrm{~A}_{8}^{\circ}-313_{7} \\
\mathrm{~B}_{7}^{\circ}-309_{8} \\
{ }^{5} \mathrm{~K}_{6}^{\circ}-278_{6}\end{array}$ & $\begin{array}{l}4111.02 \\
4110.66 \\
4109.22 \\
4108.36 \\
4105.32\end{array}$ & $\begin{array}{r}15 \\
4 \\
3 \\
30 \\
10\end{array}$ & $\begin{array}{l}24318.01 \\
24320.18 \\
24328.68 \\
24333.77 \\
24351.80\end{array}$ & $\begin{array}{r}\mathrm{A}_{7}^{\circ}-281_{7} \\
{ }^{5} \mathrm{~K}_{5}^{\circ}-249_{4} \\
\mathrm{~A}_{8}^{\circ}-319_{7} \\
{ }^{5} \mathrm{~L}_{6}^{\circ}-243_{7} \\
\mathrm{~A}_{7}^{\circ}-282_{7}\end{array}$ \\
\hline $\begin{array}{l}4222.36 \\
4219.96 \\
4218.46 \\
4216.61 \\
4215.99\end{array}$ & $\begin{array}{r}150 \\
25 \\
8 \\
6 \\
8\end{array}$ & $\begin{array}{l}23676.76 \\
23690.25 \\
23698.70 \\
23709.07 \\
23712.57\end{array}$ & $\begin{array}{r}\mathrm{A}_{7}^{\circ}-274_{8} \\
{ }^{5} \mathrm{~K}_{6}^{\circ}-279_{7} \\
\mathrm{~B}_{7}^{\circ}-310_{7} \\
{ }^{7} \mathrm{M}_{6}^{\circ}-300_{7} \\
\mathrm{D}_{7}^{\circ}-340_{7}\end{array}$ & $\begin{array}{l}4101.91 \\
4099.27 \\
4097.75 \\
4091.64 \\
4089.16\end{array}$ & $\begin{array}{r}50 \\
6 \\
15 \\
50 \\
8\end{array}$ & $\begin{array}{l}24372.03 \\
24387.75 \\
24396.79 \\
24433.24 \\
24448.02\end{array}$ & $\begin{array}{r}\mathrm{C}_{7}-324_{6} \\
{ }^{7} \mathrm{M}_{6}^{\circ}-306_{6} \\
{ }^{5} \mathrm{~K}_{5}^{\circ}-250_{5} \\
{ }^{5} \mathrm{~L}_{6}^{\circ}-244_{6} \\
{ }^{5} \mathrm{~L}_{6}^{\circ}-244_{5}\end{array}$ \\
\hline $\begin{array}{l}4215.88 \\
4215.50 \\
4211.60 \\
4209.83 \\
4207.92\end{array}$ & $\begin{array}{r}4 \\
5 \\
30 \\
3 \\
5\end{array}$ & $\begin{array}{l}23713.16 \\
23715.32 \\
23737.31 \\
23747.28 \\
23758.02\end{array}$ & $\begin{array}{r}\mathrm{A}_{8}^{\circ}-314_{7} \\
{ }^{5} \mathrm{~L}_{6}^{\circ}-237_{8} \\
{ }^{7} \mathrm{M}_{6}-299_{6} \\
{ }^{5} \mathrm{~K}_{6}^{\circ}-279_{6} \\
\mathrm{D}_{7}^{\circ}-341_{8}\end{array}$ & $\begin{array}{l}4086.68 \\
4083.04 \\
4076.63 \\
4075.48 \\
4074.62\end{array}$ & $\begin{array}{r}6 \\
6 \\
12 \\
4 \\
15\end{array}$ & $\begin{array}{l}24462.86 \\
24484.64 \\
24523.18 \\
24530.07 \\
24535.26\end{array}$ & $\begin{array}{r}\mathrm{A}_{8}^{\circ}-321_{8} \\
\mathrm{~A}_{7}^{\circ}-283_{9} \\
{ }^{5} \mathrm{~K}_{6}^{\circ}-287_{7} \\
\mathrm{~B}_{3}^{\circ}-316_{7} \\
{ }^{5} \mathrm{~L}_{6}^{\circ}-245_{5}\end{array}$ \\
\hline $\begin{array}{l}4204.16 \\
4199.63 \\
4198.22 \\
4196.40 \\
4195.62\end{array}$ & $\begin{array}{r}12 \\
10 \\
40 \\
8 \\
4\end{array}$ & $\begin{array}{l}23779.26 \\
23804.95 \\
23812.93 \\
23823.24 \\
23827.71\end{array}$ & $\begin{array}{r}{ }^{5} \mathrm{~L}_{6}^{\circ}-237_{7} \\
\mathrm{~A}_{7}^{\circ}-275_{6} \\
{ }^{5} \mathrm{~K}_{5}^{\circ}-244_{6} \\
{ }^{5} \mathrm{~K}_{6}^{\circ}-281_{6} \\
{ }^{5} \mathrm{~K}_{5}^{\circ}-244_{5}\end{array}$ & $\begin{array}{l}4073.55 \\
4072.84 \\
4070.89 \\
4068.38 \\
4066.29\end{array}$ & $\begin{array}{r}15 \\
10 \\
20 \\
4 \\
20\end{array}$ & $\begin{array}{l}24541.73 \\
24546.02 \\
24557.72 \\
24572.91 \\
24585.51\end{array}$ & $\begin{array}{r}{ }^{7} \mathrm{~K}_{5}^{\circ}-303_{4} \\
{ }^{7} \mathrm{~K}_{6}^{\circ}-315_{6} \\
{ }^{5} \mathrm{~K}_{5}^{\circ}-251_{5} \\
{ }^{7} \mathrm{~K}_{5}^{\circ}-301_{6} \\
{ }^{\circ} \mathrm{A}_{3}^{\circ}-284_{4}\end{array}$ \\
\hline $\begin{array}{l}4193.42 \\
4192.91 \\
4191.94 \\
4191.77 \\
4186.96\end{array}$ & $\begin{array}{r}5 \\
10 \\
30 \\
3 \\
30\end{array}$ & $\begin{array}{l}23840.17 \\
23843.09 \\
23848.63 \\
23849.60 \\
23876.97\end{array}$ & $\begin{array}{r}\mathrm{B}_{7}^{\circ}-311_{7} \\
{ }^{5} \mathrm{~K}_{6}^{\circ}-281_{7} \\
{ }^{5} \mathrm{~L}_{6}^{\circ}-238_{7} \\
\mathrm{C}_{7}^{\circ}-318_{7} \\
{ }^{5} \mathrm{~K}_{6}^{\circ}-282_{7}\end{array}$ & $\begin{array}{l}4064.38 \\
4064.03 \\
4061.35 \\
4060.61 \\
4059.51\end{array}$ & $\begin{array}{r}6 \\
6 \\
25 \\
4 \\
3\end{array}$ & $\begin{array}{l}24597.06 \\
24599.20 \\
24615.41 \\
24619.90 \\
24626.59\end{array}$ & $\begin{array}{r}\mathrm{B}_{7}^{\circ}-319_{8} \\
{ }^{5} \mathrm{~K}_{6}^{\circ}-289_{6} \\
{ }^{5} \mathrm{~K}_{5}^{\circ}-252_{6} \\
{ }^{5} \mathrm{~K}_{6}^{\circ}-288_{7} \\
{ }^{7} \mathrm{M}_{6}^{\circ}-307_{6}\end{array}$ \\
\hline $\begin{array}{l}4183.26 \\
4180.31 \\
4177.49 \\
4177.19 \\
4175.40\end{array}$ & $\begin{array}{r}20 \\
30 \\
4 \\
3 \\
5\end{array}$ & $\begin{array}{l}23898.08 \\
23914.97 \\
23931.12 \\
23932.82 \\
23943.09\end{array}$ & $\begin{array}{r}\mathrm{C}_{7}^{\circ}-320_{7} \\
{ }^{5} \mathrm{~K}_{5}^{\circ}-245_{5} \\
{ }^{7} \mathrm{~K}_{6}^{\circ}-309_{5} \\
{ }^{5} \mathrm{~L}_{6}^{\circ}-239_{5} \\
\mathrm{~A}_{7}^{\circ}-277_{6}\end{array}$ & $\begin{array}{l}4058.11 \\
4055.94 \\
4055.58 \\
4051.56 \\
4049.20\end{array}$ & $\begin{array}{r}30 \\
12 \\
10 \\
3 \\
15\end{array}$ & $\begin{array}{l}24635.10 \\
24648.28 \\
24650.42 \\
24674.92 \\
24689.29\end{array}$ & $\begin{array}{r}{ }^{5} \mathrm{~K}_{5}^{\circ}-252_{5} \\
\mathrm{~B}_{7}^{7}-319_{7} \\
\mathrm{~A}_{7}^{\circ}-284_{7} \\
\mathrm{~A}_{3}^{\circ}-285_{4} \\
{ }^{7} \mathrm{~K}_{5}^{\circ}-302_{6}\end{array}$ \\
\hline $\begin{array}{l}4173.68 \\
4169.85 \\
4169.06 \\
4167.04 \\
4163.75\end{array}$ & $\begin{array}{r}12 \\
5 \\
50 \\
20 \\
25\end{array}$ & $\begin{array}{l}23952.97 \\
23974.92 \\
23979.52 \\
23991.14 \\
24010.08\end{array}$ & $\begin{array}{r}\mathrm{B}_{7}-312_{8} \\
\mathrm{~B}_{7}^{\circ}-313_{7} \\
\mathrm{C}_{7}-321_{7} \\
{ }^{5} \mathrm{I}_{4}^{\circ}-284_{5} \\
{ }^{5} \mathrm{~K}_{6}^{\circ}-283_{7}\end{array}$ & $\begin{array}{l}4049.00 \\
4047.62 \\
4042.76 \\
4037.97 \\
4036.73\end{array}$ & $\begin{array}{r}3 \\
80 \\
150 \\
8 \\
3\end{array}$ & $\begin{array}{l}24690.53 \\
24698.92 \\
24728.64 \\
24757.94 \\
24765.54\end{array}$ & $\begin{array}{r}\mathrm{B}_{7}^{\circ}-320_{7} \\
{ }^{5} \mathrm{~K}_{5}^{\circ}-253_{5} \\
{ }^{5} \mathrm{~K}_{5}^{\circ}-253_{6} \\
{ }^{5} \mathrm{~K}_{6}^{\circ}-290_{6} \\
\mathrm{~A}_{7}-285_{7}\end{array}$ \\
\hline $\begin{array}{l}4162.43 \\
4160.95 \\
4160.28 \\
4157.64 \\
4156.82\end{array}$ & $\begin{array}{r}60 \\
40 \\
4 \\
8 \\
6\end{array}$ & $\begin{array}{l}24017.68 \\
24026.20 \\
24030.10 \\
24045.34 \\
24050.06\end{array}$ & $\begin{array}{r}\mathrm{A}_{7}^{\circ}-278_{8} \\
{ }^{5} \mathrm{~L}_{6}^{\circ}-240_{6} \\
{ }^{7} \mathrm{M}_{6}-302_{7} \\
{ }^{7} \mathrm{M}_{6}^{\circ}-300_{6} \\
{ }^{5} \mathrm{I}_{4}^{\circ}-285_{5}\end{array}$ & $\begin{array}{l}4035.67 \\
4035.06 \\
4034.50 \\
4031.60 \\
4025.58\end{array}$ & $\begin{array}{r}4 \\
2 \\
30 \\
5 \\
3\end{array}$ & $\begin{array}{l}24772.06 \\
24775.80 \\
24779.26 \\
24797.08 \\
24834.14\end{array}$ & $\begin{array}{r}\mathrm{B}_{7}^{\circ}-321_{7} \\
{ }^{7} \mathrm{M}_{6}^{\circ}-310_{7} \\
{ }^{5} \mathrm{I}_{4}^{\circ}-292_{5} \\
{ }^{5} \mathrm{I}_{4}^{0}-292_{4} \\
{ }^{5} \mathrm{~K}_{6}^{\circ}-291_{6}\end{array}$ \\
\hline $\begin{array}{l}4156.66 \\
4153.97 \\
4147.62 \\
4145.23 \\
4144.93\end{array}$ & $\begin{array}{r}100 \\
150 \\
3 \\
4 \\
3\end{array}$ & $\begin{array}{l}24051.04 \\
24066.58 \\
24103.46 \\
24117.34 \\
24119.09\end{array}$ & $\begin{array}{r}{ }^{5} \mathrm{~K}_{5}^{\circ}-246_{6} \\
{ }^{5} \mathrm{~L}_{6}^{\circ}-240_{7} \\
{ }^{7} \mathrm{~K}_{5}^{\circ}-298_{6} \\
\mathrm{~B}_{3}^{\circ}-312_{4} \\
\mathrm{~B}_{7}^{\circ}-314_{8}\end{array}$ & $\begin{array}{l}4022.93 \\
4013.84 \\
4008.43 \\
4005.21 \\
4004.90\end{array}$ & $\begin{array}{r}3 \\
15 \\
8 \\
80 \\
4\end{array}$ & $\begin{array}{l}24850.49 \\
24906.80 \\
24940.40 \\
24960.44 \\
24962.38\end{array}$ & $\begin{array}{r}{ }^{5} \mathrm{~K}_{6}^{\circ}-292_{6} \\
{ }^{5} \mathrm{~L}_{6}^{\circ}-249_{\mathrm{b}} \\
{ }^{7} \mathrm{~K}_{6}^{\circ}-319_{5} \\
{ }^{5} \mathrm{I}_{4}^{\circ}-294_{3} \\
{ }^{7} \mathrm{~K}_{6}^{\circ}-318_{7}\end{array}$ \\
\hline $\begin{array}{l}4141.86 \\
4139.30 \\
4137.03 \\
4136.44 \\
4133.50\end{array}$ & $\begin{array}{r}25 \\
15 \\
5 \\
10 \\
30\end{array}$ & $\begin{array}{l}24136.94 \\
24151.88 \\
24165.13 \\
24168.59 \\
24185.78\end{array}$ & $\begin{array}{r}{ }^{5} \mathrm{~K}_{5}^{\circ}-247_{4} \\
{ }^{5} \mathrm{H}_{4}^{\circ}-301_{5} \\
\mathrm{~A}_{7}^{\circ}-279_{7} \\
{ }^{5} \mathrm{~K}_{6}^{\circ}-284_{5} \\
{ }^{5} \mathrm{~L}_{8}^{\circ}-241_{7}\end{array}$ & $\begin{array}{l}4002.58 \\
4000.73 \\
3999.18 \\
3997.09 \\
3996.13\end{array}$ & $\begin{array}{r}15 \\
8 \\
40 \\
30 \\
8\end{array}$ & $\begin{array}{l}24976.82 \\
24988.39 \\
24998.08 \\
25011.18 \\
25017.18\end{array}$ & $\begin{array}{r}{ }^{5} \mathrm{I}_{4}^{\circ}-295_{3} \\
{ }^{5} \mathrm{H}_{4}^{\circ}-309_{4} \\
\mathrm{~A}_{7}^{\circ}-287_{7} \\
{ }^{7} \mathrm{~K}_{6}^{\circ}-320_{7} \\
{ }^{5} \mathrm{~L}_{6}^{\circ}-250_{5}\end{array}$ \\
\hline $\begin{array}{l}4132.02 \\
4130.66 \\
4127.34 \\
4125.78 \\
4124.03\end{array}$ & $\begin{array}{l}5 \\
8 \\
8 \\
3 \\
3\end{array}$ & $\begin{array}{l}24194.47 \\
24202.40 \\
24221.90 \\
24231.05 \\
24241.32\end{array}$ & $\begin{array}{r}{ }^{5} \mathrm{~K}_{6}^{\circ}-284_{6} \\
{ }^{7} \mathrm{M}_{6}^{\circ}-302_{6} \\
\mathrm{~A}_{7}^{\circ}-279_{6} \\
{ }^{5} \mathrm{H}_{4}^{\circ}-302_{3} \\
{ }^{7} \mathrm{M}_{6}^{\circ}-303_{6}\end{array}$ & $\begin{array}{l}3994.57 \\
3989.29 \\
3983.77 \\
3980.80 \\
3978.40\end{array}$ & $\begin{array}{r}4 \\
8 \\
10 \\
30 \\
20\end{array}$ & $\begin{array}{l}25026.96 \\
25060.05 \\
25094.77 \\
25113.51 \\
25128.64\end{array}$ & $\begin{array}{r}{ }^{7} \mathrm{M}_{8}^{\circ}-312_{7} \\
\mathrm{~A}_{7}^{\circ}-288_{6} \\
\mathrm{~A}_{7}^{\circ}-288_{7} \\
{ }^{7} \mathrm{~K}_{5}^{\circ}-307_{6}^{6} \\
\mathrm{~A}_{8}^{\circ}-327_{7}\end{array}$ \\
\hline
\end{tabular}

TABLE 4.-Classified lines of U I-Continued 
TABLE 4.-Classified lines of U $\mathrm{I}$-Continued

\begin{tabular}{|c|c|c|c|c|c|c|c|}
\hline $\begin{array}{l}3977.80 \\
3974.75 \\
3972.73 \\
3971.67 \\
3970.59\end{array}$ & $\begin{array}{r}4 \\
4 \\
3 \\
5 \\
15\end{array}$ & $\begin{array}{l}25132.42 \\
25151.75 \\
25164.54 \\
25171.26 \\
25178.08\end{array}$ & $\begin{array}{r}{ }^{7} \mathrm{~K}_{5}^{\circ}-308_{6} \\
\mathrm{~B}_{7}^{\circ}-324_{8} \\
\mathrm{~B}_{7}^{\circ}-324_{6} \\
{ }^{5} \mathrm{~K}_{5}^{\circ}-257_{6} \\
{ }^{5} \mathrm{~L}_{6}^{\circ}-251_{5}\end{array}$ & $\begin{array}{l}3847.05 \\
3846.55 \\
3845.59 \\
3843.42 \\
3842.32\end{array}$ & $\begin{array}{r}8 \\
25 \\
3 \\
8 \\
3\end{array}$ & $\begin{array}{l}25986.59 \\
25989.96 \\
25996.49 \\
26011.12 \\
26018.59\end{array}$ & $\begin{array}{r}\mathrm{C}_{7}-341_{8} \\
\mathrm{~A}_{7}^{\circ}-297_{7} \\
\mathrm{~A}_{7}^{\circ}-298_{7} \\
{ }^{5} \mathrm{~K}_{5}^{\circ}-266_{6} \\
{ }^{5} \mathrm{~K}_{6}^{\circ}-300_{6}\end{array}$ \\
\hline $\begin{array}{l}3961.98 \\
3961.52 \\
3961.42 \\
3958.93 \\
3954.10\end{array}$ & $\begin{array}{r}12 \\
40 \\
25 \\
3 \\
6\end{array}$ & $\begin{array}{l}25232.78 \\
25235.75 \\
25236.38 \\
25252.26 \\
25283.05\end{array}$ & $\begin{array}{r}\mathrm{A}_{7}^{\circ}-290_{6} \\
{ }^{5} \mathrm{~L}_{6}^{\circ}-252_{6} \\
\mathrm{~A}_{7}^{\circ}-290_{8} \\
{ }^{5} \mathrm{H}_{4}^{\circ}-313_{4} \\
{ }^{5} \mathrm{~K}_{6}^{\circ}-295_{6}\end{array}$ & $\begin{array}{l}3831.06 \\
3829.79 \\
3821.95 \\
3819.89 \\
3819.25\end{array}$ & $\begin{array}{r}5 \\
25 \\
30 \\
8 \\
6\end{array}$ & $\begin{array}{l}26095.03 \\
26103.69 \\
26157.26 \\
26171.38 \\
26175.74\end{array}$ & $\begin{array}{r}{ }^{5} \mathrm{~K}_{5}^{\circ}-267_{6} \\
{ }^{5} \mathrm{~L}_{6}^{\circ}-261_{6} \\
\mathrm{~A}_{7}^{\circ}-300_{7} \\
{ }^{5} \mathrm{~K}_{5}^{\circ}-269_{6} \\
{ }^{5} \mathrm{~K}_{6}^{\circ}-302_{6}\end{array}$ \\
\hline $\begin{array}{l}3948.65 \\
3948.45 \\
3947.50 \\
3943.82 \\
3940.09\end{array}$ & $\begin{array}{r}5 \\
35 \\
3 \\
200 \\
4\end{array}$ & $\begin{array}{l}25317.95 \\
25319.27 \\
25325.32 \\
25348.98 \\
25373.00\end{array}$ & $\begin{array}{r}{ }^{5} \mathrm{~K}_{5}^{\circ}-259_{6} \\
{ }^{5} \mathrm{~L}_{6}^{\circ}-253_{5} \\
\mathrm{~A}_{7}^{\circ}-292_{6} \\
{ }^{5} \mathrm{~L}_{6}^{\circ}-253_{6} \\
{ }^{7} \mathrm{~K}_{5}^{\circ}-311_{6}\end{array}$ & $\begin{array}{l}3801.15 \\
3800.47 \\
3797.38 \\
3791.53 \\
3789.81\end{array}$ & $\begin{array}{r}25 \\
6 \\
8 \\
4 \\
3\end{array}$ & $\begin{array}{l}26300.38 \\
26305.09 \\
26326.53 \\
26367.10 \\
26379.07\end{array}$ & $\begin{array}{r}{ }^{5} \mathrm{~K}_{5}^{\circ}-269_{5} \\
{ }^{5} \mathrm{~L}_{6}^{\circ}-263_{5} \\
{ }^{5} \mathrm{H}_{4}^{\circ}-323_{5} \\
{ }^{5} \mathrm{~K}_{6}^{\circ}-306_{7} \\
{ }^{7} \mathrm{~K}_{5}^{\circ}-321_{4}\end{array}$ \\
\hline $\begin{array}{l}3938.68 \\
3928.45 \\
3926.73 \\
3926.22 \\
3924.10\end{array}$ & $\begin{array}{r}3 \\
8 \\
30 \\
50 \\
4\end{array}$ & $\begin{array}{l}25382.08 \\
25448.16 \\
25459.33 \\
25462.62 \\
2547-6.34\end{array}$ & $\begin{array}{r}\mathrm{A}_{3}^{\circ}-292_{4} \\
\mathrm{~B}_{7}^{\circ}-327_{7} \\
{ }^{7} \mathrm{~K}_{5}^{\circ}-312_{4} \\
{ }^{5} \mathrm{~L}_{6}^{\circ}-254_{6} \\
{ }^{5} \mathrm{H}_{4}^{\circ}-314_{5}\end{array}$ & $\begin{array}{l}3788.06 \\
3781.75 \\
3779.35 \\
3778.26 \\
3775.61\end{array}$ & $\begin{array}{r}4 \\
15 \\
5 \\
8 \\
12\end{array}$ & $\begin{array}{l}26391.26 \\
26435.31 \\
26452.10 \\
26459.75 \\
26478.27\end{array}$ & $\begin{array}{r}{ }^{5} \mathrm{~L}_{6}^{\circ}-263_{7} \\
\mathrm{~A}_{3}^{\circ}-303_{4} \\
{ }^{5} \mathrm{~K}_{5}^{\circ}-270_{6} \\
\mathrm{~A}_{8}^{\circ}-341_{8} \\
\mathrm{~A}_{7}^{\circ}-3027\end{array}$ \\
\hline $\begin{array}{l}3894.12 \\
3892.26 \\
3891.50 \\
3889.11 \\
3887.10\end{array}$ & $\begin{array}{r}120 \\
3 \\
6 \\
10 \\
4\end{array}$ & $\begin{array}{l}25672.49 \\
25684.80 \\
25689.75 \\
25705.59 \\
25718.88\end{array}$ & $\begin{array}{r}{ }^{5} \mathrm{~L}_{6}^{\circ}-256_{7} \\
{ }^{5} \mathrm{~K}_{5}^{\circ}-263_{5} \\
{ }^{5} \mathrm{I}_{4}^{\circ}-301_{5} \\
{ }^{7} \mathrm{~K}_{5}^{\circ}-314_{5} \\
{ }^{7} \mathrm{Mi}_{6}^{\circ}-318_{7}\end{array}$ & $\begin{array}{l}3751.18 \\
3749.74 \\
3747.61 \\
3742.10 \\
3737.38\end{array}$ & $\begin{array}{r}35 \\
4 \\
8 \\
3 \\
5\end{array}$ & $\begin{array}{l}26650.74 \\
26660.94 \\
26676.10 \\
26715.37 \\
26749.10\end{array}$ & $\begin{array}{r}\mathrm{A}_{7}^{\circ}-302_{6} \\
{ }^{5} \mathrm{~K}_{6}^{\circ}-309_{5} \\
{ }^{5} \mathrm{I}_{4}^{\circ}-311_{5} \\
{ }^{5} \mathrm{~L}_{6}^{\circ}-267_{6} \\
{ }^{5} \mathrm{~K}_{6}^{\circ}-310_{7}\end{array}$ \\
\hline $\begin{array}{l}3886.14 \\
3885.99 \\
3881.15 \\
3879.53 \\
3876.45\end{array}$ & $\begin{array}{r}4 \\
8 \\
4 \\
30 \\
5\end{array}$ & $\begin{array}{l}25725.18 \\
25726.18 \\
25758.31 \\
25769.02 \\
25789.50\end{array}$ & $\begin{array}{r}{ }^{7} \mathrm{M}_{6}-319_{7} \\
{ }^{7} \mathrm{~K}_{5}^{\circ}-315_{5} \\
\mathrm{~A}_{7}^{\circ}-295_{6} \\
{ }^{5} \mathrm{I}_{4}-302_{3} \\
{ }^{7} \mathrm{~K}_{5}^{\circ}-315_{6}\end{array}$ & $\begin{array}{l}3736.02 \\
3733.17 \\
3732.26 \\
3731.45 \\
3725.31\end{array}$ & $\begin{array}{r}12 \\
3 \\
60 \\
100 \\
6\end{array}$ & $\begin{array}{l}26758.88 \\
26779.28 \\
26785.86 \\
26791.64 \\
26835.81\end{array}$ & $\begin{array}{r}{ }^{5} \mathrm{~L}_{6}-268_{6} \\
\mathrm{~B}_{7}-341_{8} \\
\mathrm{~A}_{7}-305_{6} \\
{ }^{5} \mathrm{~L}_{6}^{\circ}-269_{6} \\
\mathrm{~A}_{7}^{\circ}-306_{6}\end{array}$ \\
\hline $\begin{array}{l}3876.13 \\
3873.08 \\
3871.04 \\
3867.50 \\
3867.17\end{array}$ & $\begin{array}{r}60 \\
15 \\
120 \\
15 \\
30\end{array}$ & $\begin{array}{l}25791.63 \\
25811.98 \\
25825.59 \\
25849.18 \\
25851.41\end{array}$ & $\begin{array}{r}{ }^{5} \mathrm{~L}_{6}-257_{6} \\
\mathrm{~A}_{7}^{\circ}-296_{8} \\
{ }^{5} \mathrm{~L}_{6}^{\circ}-258_{6} \\
{ }^{7} \mathrm{M}_{6}^{\circ}-321_{7} \\
\mathrm{~A}_{3}^{\circ}-297_{4}\end{array}$ & $\begin{array}{l}3724.45 \\
3719.29 \\
3718.31 \\
3717.72 \\
3713.56\end{array}$ & $\begin{array}{r}15 \\
40 \\
6 \\
20 \\
20\end{array}$ & $\begin{array}{l}26842.02 \\
26879.22 \\
26886.30 \\
26890.55 \\
26920.71\end{array}$ & $\begin{array}{r}\mathrm{A}_{7}^{\circ}-306_{7} \\
{ }^{5} \mathrm{~K}_{5}^{\circ}-274_{8} \\
{ }^{5} \mathrm{I}_{4}^{\circ}-313_{5} \\
{ }^{5} \mathrm{~K}_{6}^{\circ}-311_{7} \\
{ }^{5} \mathrm{~L}_{6}^{\circ}-269_{5}\end{array}$ \\
\hline $\begin{array}{l}3864.10 \\
3855.43 \\
3854.22 \\
3852.97 \\
3851.72\end{array}$ & $\begin{array}{r}30 \\
20 \\
100 \\
5 \\
20\end{array}$ & $\begin{array}{l}25871.93 \\
25930.12 \\
25938.25 \\
25946.65 \\
25955.07\end{array}$ & $\begin{array}{r}{ }^{7} \mathrm{~K}_{5}^{\circ}-316_{4} \\
{ }^{5} \mathrm{~K}_{5}^{\circ}-265_{6} \\
{ }^{5} \mathrm{~L}_{6}^{\circ}-259_{6} \\
{ }^{5} \mathrm{~K}_{5}-265_{5} \\
{ }^{5} \mathrm{H}_{4}^{\circ}-319_{5}\end{array}$ & $\begin{array}{l}3707.95 \\
3704.65 \\
3703.27 \\
3702.62 \\
3699.18\end{array}$ & $\begin{array}{r}25 \\
3 \\
30 \\
25 \\
10\end{array}$ & $\begin{array}{l}26961.41 \\
26985.45 \\
26995.50 \\
27000.26 \\
27025.36\end{array}$ & $\begin{array}{r}\mathrm{B}_{3}^{\circ}-340_{2} \\
{ }^{5} \mathrm{~K}_{5}^{\circ}-275_{6} \\
{ }^{5} \mathrm{~K}_{5}^{\circ}-276_{6} \\
{ }^{5} \mathrm{~K}_{6}^{\circ}-312_{7} \\
{ }^{5} \mathrm{~K}_{6}^{\circ}-313_{7}\end{array}$ \\
\hline
\end{tabular}

TABLE 4.-Classified lines of $\mathrm{U} \mathrm{I}$-Continued 


\begin{tabular}{|c|c|c|c|c|c|c|c|}
\hline$\lambda_{\mathrm{air}} \mathrm{A}$ & Intensity & $\nu_{\mathrm{Vac}} \mathrm{cm}^{-1}$ & Term combination & $\lambda_{\mathrm{air}} \mathrm{A}$ & Intensity & $\nu_{\mathrm{vac}} \mathrm{cm}^{-1}$ & Term combination \\
\hline $\begin{array}{l}3695.21 \\
3694.17 \\
3692.75 \\
3692.43 \\
3689.86\end{array}$ & $\begin{array}{r}20 \\
5 \\
30 \\
10 \\
5\end{array}$ & $\begin{array}{l}27054.42 \\
27062.01 \\
27072.40 \\
27074.79 \\
27093.65\end{array}$ & $\begin{array}{r}{ }^{7} \mathrm{~K}_{6}^{\circ}-340_{7} \\
{ }^{5} \mathrm{~K}_{5}^{\circ}-276_{5} \\
{ }^{5} \mathrm{~L}_{6}^{\circ}-270_{6} \\
\mathrm{~A}_{7}^{\circ}-307_{6} \\
\mathrm{~A}_{7}^{\circ}-308_{6}\end{array}$ & $\begin{array}{l}3548.42 \\
3546.55 \\
3543.21 \\
3542.57 \\
3540.00\end{array}$ & $\begin{array}{r}6 \\
25 \\
4 \\
25 \\
5\end{array}$ & $\begin{array}{l}28173.57 \\
28188.41 \\
28214.98 \\
28220.04 \\
28240.59\end{array}$ & $\begin{array}{r}\mathrm{A}_{7}^{\circ}-319_{7} \\
{ }^{5} \mathrm{~L}_{6}^{\circ}-281_{5} \\
{ }^{5} \mathrm{~K}_{6}^{\circ}-324_{6} \\
{ }^{5} \mathrm{~K}_{6}^{\circ}-325_{6} \\
{ }^{5} \mathrm{~K}_{5}^{\circ}-288_{6}\end{array}$ \\
\hline $\begin{array}{l}3687.46 \\
3685.78 \\
3679.38 \\
3677.39 \\
3676.52\end{array}$ & $\begin{array}{l}30 \\
40 \\
60 \\
60 \\
10\end{array}$ & $\begin{array}{l}27111.26 \\
27123.64 \\
27170.82 \\
27185.47 \\
27191.91\end{array}$ & $\begin{array}{r}\mathrm{A}_{3}^{\circ}-309_{4} \\
{ }^{5} \mathrm{~K}_{5}^{\circ}-277_{6} \\
{ }^{5} \mathrm{~K}_{5}^{\circ}-278_{5} \\
\mathrm{~A}_{7}^{\circ}-309_{8} \\
{ }^{5} \mathrm{~K}_{6}^{\circ}-314_{5}\end{array}$ & $\begin{array}{l}3538.23 \\
3535.96 \\
3534.33 \\
3532.89 \\
3518.47\end{array}$ & $\begin{array}{r}4 \\
10 \\
30 \\
8 \\
5\end{array}$ & $\begin{array}{l}28254.66 \\
28272.78 \\
28285.84 \\
28297.41 \\
28413.33\end{array}$ & $\begin{array}{r}{ }^{5} \mathrm{~K}_{5}^{\circ}-289_{6} \\
\mathrm{~A}_{3}^{\circ}-321_{4} \\
{ }^{5} \mathrm{~L}_{6}^{\circ}-283_{7} \\
\mathrm{~A}_{7}^{\circ}-321_{7} \\
{ }^{5} \mathrm{~K}_{5}^{\circ}-290_{6}\end{array}$ \\
\hline $\begin{array}{l}3673.73 \\
3672.19 \\
3665.21 \\
3663.19 \\
3659.16\end{array}$ & $\begin{array}{r}6 \\
5 \\
12 \\
8 \\
100\end{array}$ & $\begin{array}{l}27212.54 \\
27223.97 \\
27275.84 \\
27290.84 \\
27320.93\end{array}$ & $\begin{array}{r}{ }^{5} \mathrm{~K}_{6}^{\circ}-315_{5} \\
\mathrm{~A}_{7}^{\circ}-310_{7} \\
{ }^{5} \mathrm{~K}_{6}^{\circ}-315_{6} \\
{ }^{5} \mathrm{I}_{4}^{\circ}-317_{5} \\
{ }^{5} \mathrm{~K}_{5}^{\circ}-278_{6}\end{array}$ & $\begin{array}{l}3514.61 \\
3511.44 \\
3509.06 \\
3507.34 \\
3507.05\end{array}$ & $\begin{array}{r}200 \\
30 \\
8 \\
80 \\
30\end{array}$ & $\begin{array}{l}28444.54 \\
28470.23 \\
28489.58 \\
28503.50 \\
28505.87\end{array}$ & $\begin{array}{r}{ }^{5} \mathrm{~L}_{6}^{\circ}-284_{5} \\
{ }^{5} \mathrm{~L}_{6}^{\circ}-284_{6} \\
{ }^{5} \mathrm{~K}_{5}^{\circ}-291_{6} \\
{ }^{5} \mathrm{~L}_{6}^{\circ}-285_{5} \\
{ }^{5} \mathrm{~K}_{5}^{\circ}-292_{6}\end{array}$ \\
\hline $\begin{array}{l}3658.68 \\
3654.89 \\
3653.21 \\
3651.93 \\
3651.04\end{array}$ & $\begin{array}{r}12 \\
40 \\
25 \\
15 \\
4\end{array}$ & $\begin{array}{l}27324.51 \\
27352.83 \\
27365.41 \\
27375.04 \\
27381.68\end{array}$ & $\begin{array}{r}5 \mathrm{~L}_{6}^{\circ}-273_{7} \\
\mathrm{~A}_{3}^{\circ}-312_{4} \\
\mathrm{~A}_{7}^{\circ}-311_{7} \\
\mathrm{~A}_{3}^{\circ}-313_{4} \\
{ }^{5} \mathrm{~L}_{6}^{\circ}-273_{5}\end{array}$ & $\begin{array}{l}3500.07 \\
3499.61 \\
3493.99 \\
3489.37 \\
3486.12\end{array}$ & $\begin{array}{r}80 \\
4 \\
40 \\
80 \\
3\end{array}$ & $\begin{array}{l}28562.71 \\
28566.46 \\
28612.42 \\
28650.33 \\
28677.02\end{array}$ & $\begin{array}{r}{ }^{5} \mathrm{~L}_{6}^{\circ}-286_{5} \\
{ }^{5} \mathrm{~L}_{6}^{\circ}-285_{7} \\
{ }^{5} \mathrm{~K}_{5}^{\circ}-292_{5} \\
{ }^{5} \mathrm{~L}_{6}^{\circ}-287_{5} \\
\mathrm{~A}_{7}^{\circ}-324_{8}\end{array}$ \\
\hline $\begin{array}{l}3649.86 \\
3648.25 \\
3644.24 \\
3639.49 \\
3638.60\end{array}$ & $\begin{array}{r}3 \\
15 \\
60 \\
30 \\
3\end{array}$ & $\begin{array}{l}27390.55 \\
27402.64 \\
27432.77 \\
27468.58 \\
27475.25\end{array}$ & $\begin{array}{r}{ }^{7} \mathrm{M}_{6}^{\circ}-336_{6} \\
{ }^{5} \mathrm{~K}_{5}^{\circ}-279_{6} \\
{ }^{5} \mathrm{~K}_{5}^{\circ}-280_{6} \\
{ }^{5} \mathrm{~K}_{6}^{\circ}-317_{5} \\
\mathrm{~A}_{7}^{\circ}-312_{7}\end{array}$ & $\begin{array}{l}3473.56 \\
3466.30 \\
3463.90 \\
3462.21 \\
3454.61\end{array}$ & $\begin{array}{r}8 \\
40 \\
8 \\
30 \\
30\end{array}$ & $\begin{array}{l}28780.70 \\
28840.99 \\
28860.94 \\
28875.01 \\
28938.53\end{array}$ & $\begin{array}{r}{ }^{5} \mathrm{~K}_{5}^{\circ}-294_{6} \\
\mathrm{~A}_{7}^{\circ}-326_{7} \\
{ }^{5} \mathrm{~L}_{6}^{\circ}-288_{6} \\
{ }^{5} \mathrm{~L}_{6}^{\circ}-289_{6} \\
{ }^{5} \mathrm{~K}_{5}^{\circ}-295_{6}\end{array}$ \\
\hline $\begin{array}{l}3638.20 \\
3636.31 \\
3635.30 \\
3627.70 \\
3626.36\end{array}$ & $\begin{array}{r}150 \\
15 \\
30 \\
6 \\
15\end{array}$ & $\begin{array}{l}27478.35 \\
27492.60 \\
27500.27 \\
27557.85 \\
27568.05\end{array}$ & $\begin{array}{r}\mathrm{A}_{7}^{\circ}-312_{8} \\
{ }^{5} \mathrm{I}_{4}^{\circ}-319_{5} \\
\mathrm{~A}_{7}^{\circ}-313_{7} \\
\mathrm{~A}_{7}^{\circ}-314_{7} \\
{ }^{5} \mathrm{~K}_{5}^{\circ}-281_{5}\end{array}$ & $\begin{array}{l}3435.49 \\
3434.28 \\
3432.36 \\
3431.14 \\
3418.39\end{array}$ & $\begin{array}{r}20 \\
10 \\
4 \\
8 \\
5\end{array}$ & $\begin{array}{l}29099.60 \\
29109.85 \\
29126.15 \\
29136.53 \\
29245.18\end{array}$ & $\begin{array}{r}{ }^{5} \mathrm{~K}_{5}^{\circ}-297_{4} \\
{ }^{5} \mathrm{~L}_{6}^{\circ}-291_{6} \\
{ }^{5} \mathrm{~L}_{6}^{\circ}-292_{6} \\
{ }^{5} \mathrm{~K}_{6}^{\circ}-334_{6} \\
{ }^{5} \mathrm{~K}_{5}^{\circ}-298_{6}\end{array}$ \\
\hline $\begin{array}{l}3620.08 \\
3616.33 \\
3612.96 \\
3611.40 \\
3603.74\end{array}$ & $\begin{array}{r}60 \\
40 \\
5 \\
20 \\
25\end{array}$ & $\begin{array}{l}27615.81 \\
27644.47 \\
27670.29 \\
27682.26 \\
27741.03\end{array}$ & $\begin{array}{r}{ }^{5} \mathrm{~L}_{6}^{\circ}-276_{6} \\
\mathrm{~A}_{7}^{\circ}-314_{8} \\
{ }^{5} \mathrm{~K}_{6}^{\circ}-319_{5} \\
{ }^{5} \mathrm{~L}_{6}^{\circ}-276_{5} \\
{ }^{5} \mathrm{~K}_{6}^{\circ}-320_{7}\end{array}$ & $\begin{array}{l}3412.58 \\
3404.33 \\
3400.28 \\
3386.24 \\
3376.04\end{array}$ & $\begin{array}{r}5 \\
12 \\
2 \\
5 \\
4\end{array}$ & $\begin{array}{l}29295.00 \\
29366.00 \\
29400.95 \\
29522.83 \\
29612.00\end{array}$ & $\begin{array}{r}{ }^{5} \mathrm{~K}_{6}^{\circ}-335_{7} \\
{ }^{5} \mathrm{~K}_{5}^{\circ}-299_{6} \\
{ }^{5} \mathrm{~L}_{6}^{\circ}-294_{6} \\
{ }^{5} \mathrm{~K}_{5}^{\circ}-301_{5} \\
{ }^{5} \mathrm{I}_{4}^{\circ}-340_{3}\end{array}$ \\
\hline $\begin{array}{l}3603.36 \\
3602.48 \\
3600.56 \\
3598.94 \\
3594.81\end{array}$ & $\begin{array}{r}30 \\
30 \\
3 \\
30 \\
8\end{array}$ & $\begin{array}{l}27743.96 \\
27750.75 \\
27765.55 \\
27778.02 \\
27810.01\end{array}$ & $\begin{array}{r}{ }^{5} \mathrm{~L}_{6}^{\circ}-277_{6} \\
\mathrm{~A}_{7}^{\circ}-315_{6} \\
\mathrm{~A}_{3}^{\circ}-316_{4} \\
{ }^{5} \mathrm{~L}_{6}^{\circ}-277_{5} \\
{ }^{5} \mathrm{~K}_{5}^{\circ}-283_{4}\end{array}$ & $\begin{array}{l}3368.98 \\
3358.14 \\
3250.51 \\
3347.37 \\
3345.89\end{array}$ & $\begin{array}{r}5 \\
8 \\
8 \\
5 \\
15\end{array}$ & $\begin{array}{l}29674.05 \\
29769.87 \\
29837.69 \\
29865.62 \\
29878.88\end{array}$ & $\begin{array}{r}{ }^{5} \mathrm{~K}_{5}^{\circ}-300_{6} \\
\mathrm{~A}_{7}^{\circ}-335_{7} \\
{ }^{5} \mathrm{~L}_{6}^{\circ}-299_{7} \\
{ }^{5} \mathrm{~L}_{6}^{\circ}-298_{6} \\
{ }^{5} \mathrm{~K}_{5}^{\circ}-304_{4}\end{array}$ \\
\hline $\begin{array}{l}3593.20 \\
3592.97 \\
3591.74 \\
3589.66 \\
3587.78\end{array}$ & $\begin{array}{r}40 \\
15 \\
50 \\
20 \\
4\end{array}$ & $\begin{array}{l}27822.47 \\
27824.23 \\
27833.72 \\
27849.84 \\
27864.45\end{array}$ & $\begin{array}{r}{ }^{5} \mathrm{~K}_{6}^{\circ}-321_{7} \\
{ }^{5} \mathrm{~K}_{5}^{\circ}-284_{5} \\
{ }^{5} \mathrm{~K}_{5}^{\circ}-284_{4} \\
{ }^{5} \mathrm{~K}_{5}^{\circ}-284_{6} \\
{ }^{5} \mathrm{I}_{4}^{\circ}-323_{5}\end{array}$ & $\begin{array}{l}3337.03 \\
3333.89 \\
3316.55 \\
3310.63 \\
3303.84\end{array}$ & $\begin{array}{r}12 \\
8 \\
8 \\
6 \\
8\end{array}$ & $\begin{array}{l}29958.16 \\
29986.42 \\
30143.21 \\
30197.08 \\
30259.13\end{array}$ & $\begin{array}{l}{ }^{5} \mathrm{~L}_{6}^{\circ}-300_{7} \\
{ }^{5} \mathrm{~L}_{6}^{\circ}-299_{6} \\
{ }^{5} \mathrm{~L}_{6}^{\circ}-301_{5} \\
\mathbf{A}_{3}-340_{3} \\
\mathbf{A}_{7}^{\circ}-340_{7}\end{array}$ \\
\hline $\begin{array}{l}3585.38 \\
3584.88 \\
3580.25 \\
3577.92 \\
3577.78\end{array}$ & $\begin{array}{r}25 \\
250 \\
25 \\
30 \\
8\end{array}$ & $\begin{array}{l}27883.15 \\
27887.01 \\
27923.08 \\
27941.25 \\
27942.31\end{array}$ & $\begin{array}{r}{ }^{5} \mathrm{~K}_{5}-285_{5} \\
{ }^{5} \mathrm{~L}_{6}-278_{7} \\
{ }^{5} \mathrm{~K}_{5}^{\circ}-285_{4} \\
{ }^{5} \mathrm{~L}_{6}^{\circ}-278_{6} \\
{ }^{5} \mathrm{~K}_{5}^{\circ}-286_{5}\end{array}$ & $\begin{array}{l}3301.65 \\
3298.88 \\
3292.94 \\
3282.98 \\
3278.79\end{array}$ & $\begin{array}{r}4 \\
8 \\
15 \\
2 \\
3\end{array}$ & $\begin{array}{l}30279.21 \\
30304.58 \\
30359.30 \\
30451.43 \\
30490.29\end{array}$ & $\begin{array}{r}{ }^{5} \mathrm{~L}_{6}^{\circ}-302_{7} \\
\mathrm{~A}_{7}^{\circ}-341_{8} \\
{ }^{5} \mathrm{~K}_{5}^{\circ}-309_{4} \\
{ }_{5}^{5} \mathrm{~L}_{6}^{\circ}-302_{6} \\
{ }^{5} \mathrm{~L}_{6}^{\circ}-303_{6}\end{array}$ \\
\hline $\begin{array}{l}3574.76 \\
3567.49 \\
3566.60 \\
3565.05 \\
3564.27\end{array}$ & $\begin{array}{r}50 \\
4 \\
200 \\
10 \\
3\end{array}$ & $\begin{array}{l}27965.94 \\
28022.92 \\
28029.97 \\
28042.15 \\
28048.26\end{array}$ & $\begin{array}{r}{ }^{5} \mathrm{~L}_{6}-279_{7} \\
{ }^{5} \mathrm{~L}_{6}-279_{6} \\
{ }^{5} \mathrm{~K}_{5}^{\circ}-287_{5} \\
{ }^{5} \mathrm{~K}_{6}^{\circ}-323_{5} \\
{ }^{5} \mathrm{~L}_{6}^{\circ}-280_{7}\end{array}$ & $\begin{array}{l}3277.73 \\
3276.76 \\
3276.15 \\
3268.45 \\
3266.93\end{array}$ & $\begin{array}{r}6 \\
10 \\
5 \\
4 \\
20\end{array}$ & $\begin{array}{l}30500.20 \\
30509.19 \\
30514.84 \\
30586.72 \\
30600.98\end{array}$ & $\begin{array}{r}{ }^{5} \mathrm{~L}_{6}^{\circ}-304_{6} \\
{ }^{5} \mathrm{~K}_{5}^{\circ}-311_{5} \\
{ }^{5} \mathrm{~K}_{5}^{\circ}-311_{6} \\
{ }^{5} \mathbf{L}_{6}^{\circ}-305_{6} \\
{ }^{5} \mathrm{~K}_{5}^{\circ}-312_{4}\end{array}$ \\
\hline $\begin{array}{l}3563.66 \\
3557.84 \\
3555.32 \\
3554.88 \\
3549.20\end{array}$ & $\begin{array}{r}50 \\
50 \\
60 \\
8 \\
25\end{array}$ & $\begin{array}{l}28053.05 \\
28098.91 \\
28118.85 \\
28122.37 \\
28167.36\end{array}$ & $\begin{array}{l}{ }^{5} \mathrm{~L}_{6}^{\circ}-280_{6} \\
{ }^{5} \mathrm{~L}_{6}^{\circ}-281_{6} \\
{ }^{5} \mathrm{~L}_{6}^{\circ}-281_{7} \\
\mathrm{~A}_{7}^{\circ}-319_{8} \\
\mathrm{~A}_{7}^{\circ}-318_{7}\end{array}$ & $\begin{array}{l}3264.55 \\
3263.12 \\
3254.33 \\
3238.67 \\
3237.87\end{array}$ & $\begin{array}{r}6 \\
20 \\
8 \\
2 \\
15\end{array}$ & $\begin{array}{l}30623.30 \\
30636.75 \\
30719.49 \\
30868.01 \\
30875.62\end{array}$ & $\begin{array}{r}{ }^{5} \mathrm{~K}_{5}^{\circ}-313_{4} \\
{ }^{5} \mathrm{~L}_{6}^{\circ}-306_{6} \\
{ }^{5} \mathrm{~K}_{5}^{\circ}-313_{5} \\
{ }^{5} \mathrm{~K}_{5}^{\circ}-315_{5} \\
{ }^{5} \mathrm{~L}_{6}^{\circ}-307_{6}\end{array}$ \\
\hline
\end{tabular}


TABLE 4.-Classified lines of $\mathrm{U}_{\mathrm{I}}$-Continued

\begin{tabular}{|c|c|c|c|}
\hline$\lambda_{\text {air } \mathrm{A}}$ & Intensity & $\nu_{\mathbf{V} \mathbf{a c}} \mathrm{Cm}^{-1}$ & Term combination \\
\hline 3235.90 & 6 & 30894.46 & ${ }^{5} \mathrm{~L}_{6}^{\circ}-308_{6}$ \\
\hline 3225.60 & 2 & 30993.09 & ${ }^{5} \mathrm{~L}_{6}^{0}-310_{5}$ \\
\hline 3223.45 & 8 & 31013.72 & ${ }^{5} \bar{K}_{5}^{\circ}-316_{4}$ \\
\hline 3212.03 & 8 & 31124.03 & ${ }^{5} \mathrm{~K}_{5}^{0}-317_{5}$ \\
\hline 3211.46 & 2 & 31129.52 & ${ }^{5} \mathrm{~L}_{6}^{\circ}-311_{5}$ \\
\hline 3210.90 & 5 & 31134.96 & ${ }^{5} \mathrm{~L}_{6}-311_{6}$ \\
\hline 3207.67 & 6 & 31166.29 & ${ }^{5} \mathrm{~L}_{6}^{0}-311_{7}$ \\
\hline 3196.41 & 3 & 31276.12 & ${ }^{5} \mathrm{~L}_{6}^{0}-312_{7}$ \\
\hline 3189.91 & 5 & 31339.84 & ${ }^{5} \mathrm{~L}_{6}^{\circ}-313_{5}$ \\
\hline 3187.99 & 8 & 31358.73 & ${ }^{5} \mathrm{~L}_{6}^{\circ}-314_{7}$ \\
\hline 3174.87 & 3 & 31488.25 & ${ }^{5} \mathrm{~L}_{6} \cdot-315_{5}$ \\
\hline 3171.58 & 5 & 31520.94 & ${ }^{5} \mathrm{~K}_{5}^{0}-321_{4}$ \\
\hline 3136.30 & 4 & 31875.54 & ${ }^{5} \mathrm{~K}_{5}^{\circ}-325_{6}$ \\
\hline 3129.38 & 3 & 31946.01 & ${ }^{5} \mathrm{~L}_{6}^{0}-319_{5}$ \\
\hline 3122.46 & 4 & 32016.76 & ${ }^{5} \mathrm{~L}_{6}^{\circ}-320_{7}$ \\
\hline 3114.54 & 4 & 32098.19 & ${ }^{5} \mathrm{~L}_{6}-321_{7}$ \\
\hline 3076.91 & 2 & 32490.73 & ${ }^{5} \mathrm{~L}_{6}^{\circ}-324_{6}$ \\
\hline 3076.43 & 8 & 32495.83 & ${ }^{5} \mathrm{~L}_{6}^{\circ}-325_{6}$ \\
\hline 3062.66 & 2 & 32641.92 & ${ }^{5} \mathrm{~L}_{6}^{0}-326_{7}$ \\
\hline 3048.64 & 3 & 32791.96 & ${ }^{5} \mathrm{~K}_{5}^{0}-334_{6}$ \\
\hline 3027.66 & 5 & 33019.20 & ${ }^{5} \mathrm{~K}_{5}^{\circ}-336_{6}$ \\
\hline 2992.04 & 3 & 33412.29 & ${ }^{5} \mathrm{~L}_{6}^{\circ}-334_{6}$ \\
\hline 2977.92 & 8 & 33570.67 & ${ }^{5} \mathrm{~L}_{6}^{\circ}-335_{7}$ \\
\hline 2671.82 & $\begin{array}{l}8 \\
6\end{array}$ & 33639.60 & $\begin{array}{l}{ }^{5} \mathrm{~L}_{6}^{\circ}-336_{6} \\
{ }^{5} \mathrm{~L}_{0}-340\end{array}$ \\
\hline 2935.15 & & 34059.87 & ${ }^{5} \mathrm{~L}_{6}^{6}-3$ \\
\hline
\end{tabular}

From the spectroscopic evidence presented in this paper, it is clear that uranium is an element of the rare-earth type; that, in fact, it is the homologue of neodymium, being the third member of a series beginning with thorium. That the seventh period of elements in the periodic system should encompass a second group of rare earths similar to the group in the sixth period was foreseen more than 20 years ago by Bohr [14]. Since that time opinion has differed concerning the atomic number at which the binding of the $5 f$ electron first sets in. Some investigators, notably Villar [15], have suggested $\mathrm{Th}(90)$, others $\mathrm{U}(92)$, and still others, including Starke [16], the transuranium element 93 as the first in which a $5 f$ electron appears. In each instance the basis of opinion was evidence derived from chemical behavior, nuclear activity, behavior of ions in crystals and solutions, etc. The testimony of the spectroscope, however, in selecting thorium for this role is unique and decisive, and it is supported by the recent spatial classifications of the elements by Djounkovsky and Kavos [17] and by Talpain [18], which array thorium, protoactinium, and uranium as the chemical homologues of cerium, praseodymium, and neodymium.

At the present time the state of the analysis of U I does not permit a calculation to be made of the ionization potential of neutral uranium atoms from series-forming terms. Nevertheless, an approximate value of 4 volts for this important atomic constant may be estimated from the fact that no lines of neutral atoms have been found below wavelength $2900 \mathrm{~A}$. This accounts for the ease with which uranium is ionized in electric arcs, and magnetic fields, and explains the appearance of lines due to ionized atoms in the red and near infrared regions and their overwhelmimg preponderance in the regions of shorter wavelength.

\section{References}

[1] H. Kayser, Handbuch der Spectroscopie, p. 734 (S. Hirzel, Leipzig, 1912).

[2] K. W. Meissner, Ann. Physik 50, 713 (1916).

[3] C. C. Kiess and W. F. Meggers, BS Sci. Pap. 16, 51 (1920) S372.

[4] G. R. Harrison, MIT wavelength tables (John Wiley \& Sons, Inc., New York, N. Y., 1939).

[5] W. J. Humphreys, Astrophys, J. 6, 169 (1897).

[6] J. C. McLennan, E. Cohen, M. J. Liggett, Trans. Roy. Soc. Can. 20, 365 (1926).

[7] C. C. Kiess and E. Z. Stowell, BS J. Research 12, 460 (1934) RP671.

[8] R. W. Wood, Nature 140, 723 (1937).

[9] W. F. Meggers and K. Burns, BS Sci. Pap. 18, 201 (1922) S442.
[10] W. F. Meggers and O. Laporte, Phys. Rev. 28, 642 (1926).

[11] E. Back, Ann. Physik. [IV] 70, 333 (1923).

[12] J. R. McNally, G. R. Harrison, and H. B. Park, J. Opt. Soc. Am. 32, 334 (1942); T. L. de Bruin, P. Schuurmans, and P. F. A. Klinkenberg, Z. Physik 121, 667 (1943) ; 122, 23, (1944).

[13] O. Laporte and J. E. Mack, Phys. Rev. 63, 246 (1943).

[14] N. Bohr, Nature 112, 43 (1923).

[15] G. E. Villar; Ann. acad. brasil. sci. 12, 51, (1940).

[16] K. Starke, Z. anorg. allgem. Chem. 251, 251 (1943).

[17] G. E. Djounkovsky and S. Kavos, J. phys. radium [8] 5, 53 (1944).

[18] L. Talpain, J. phys. radium [8] 6, 176 (1945).

Washington, April 30, 1946. 